\title{
Mathematical Model of MDR-TB and XDR-TB with Isolation and Lost to Follow-Up
}

\author{
F. B. Agusto, J. Cook, P. D. Shelton, and M. G. Wickers \\ Department of Mathematics and Statistics, Austin Peay State University, Clarksville, TN 37044, USA \\ Correspondence should be addressed to F. B. Agusto; fbagusto@gmail.com
}

Received 24 February 2015; Accepted 31 May 2015

Academic Editor: Juan-Carlos Cortés

Copyright (C) 2015 F. B. Agusto et al. This is an open access article distributed under the Creative Commons Attribution License, which permits unrestricted use, distribution, and reproduction in any medium, provided the original work is properly cited.

\begin{abstract}
We present a deterministic model with isolation and lost to follow-up for the transmission dynamics of three strains of Mycobacterium tuberculosis (TB), namely, the drug sensitive, multi-drug-resistant (MDR), and extensively-drug-resistant (XDR) TB strains. The model is analyzed to gain insights into the qualitative features of its associated equilibria. Some of the theoretical and epidemiological findings indicate that the model has locally asymptotically stable (LAS) disease-free equilibrium when the associated reproduction number is less than unity. Furthermore, the model undergoes in the presence of disease reinfection the phenomenon of backward bifurcation, where the stable disease-free equilibrium of the model coexists with a stable endemic equilibrium when the associated reproduction number is less than unity. Further analysis of the model indicates that the diseasefree equilibrium is globally asymptotically stable (GAS) in the absence of disease reinfection. The result of the global sensitivity analysis indicates that the dominant parameters are the disease progression rate, the recovery rate, the infectivity parameter, the isolation rate, the rate of lost to follow-up, and fraction of fast progression rates. Our results also show that increase in isolation rate leads to a decrease in the total number of individuals who are lost to follow-up.
\end{abstract}

\section{Introduction}

Mycobacterium tuberculosis (TB) is caused by bacteria that are transmitted from person to person through the air by an infected person's coughing, sneezing, speaking, or singing [1]. TB usually affects the lungs, but it can also affect other parts of the body, such as the brain, the kidneys, or the spine [1]. The TB bacteria can stay in the air for several hours, depending on the environment. In 2013, 9 million people were ill with TB, and 1.5 million mortalities occurred from the disease [2]; over $95 \%$ of deaths occurred in low- and middle-income countries [2]. About one-third of the world's population has latent TB [2]. TB is also among the top three causes of death in women aged 15 to 44 [2]. Tuberculosis is second only to HIV/AIDS as the greatest killer worldwide due to a single infectious agent [2]. Those who have a compromised immune system, like those who are living with HIV, malnutrition, or diabetes, or people who use tobacco products, have a much higher risk of falling ill. Individuals who develop TB are provided with a six-month course of four antimicrobial drugs along with supervision and support by a health worker. Improper treatment compliance or use of poor quality medicines can all lead to the development of drug-resistant tuberculosis [2].

Multi-drug-resistant (MDR) TB is a form of TB caused by bacteria that do not respond to, at least, isoniazid and rifampicin, which are the two most powerful, standard antituberculosis drugs. MDR-TB is treatable and curable by using second-line drugs [3]. However, these treatment options are limited and recommended medicines are not always available [3]. In some cases, more severe drug resistance can develop. Extensively-drug-resistant (XDR) TB is a form of multi-drugresistant TB that responds to even fewer available medicines, including the second-line drugs [3]. In 2013, there were about 480,000 cases of MDR-TB present in the world [4]; it was estimated that about $9 \%-10 \%$ of these cases were XDR-TB $[2,4-6]$.

The increase in drug-resistant TB strains has called for an increased urgency for isolating individuals infected with such strains of TB [7]. High priority is being placed on identifying and curing these individuals. With proper identification and treatment, about $40 \%$ of XDR-TB cases could potentially be cured $[6,8]$. However, only $10 \%$ of MDR-TB cases are ever 
identified, leaving the potential development of XDR-TB to become more prevalent worldwide [2]. The need for a modern and effective approach to curtail the rise of drug-resistant TB strains is being sought after, one of which is constructed isolation, whether it is an at-home isolation or isolation at a medical facility [6]. An event that portrays the need for carefully constructed isolation was the identification of an individual infected with MDR-TB in Atlanta International Airport in 2007 [9]. The individual had flown to Atlanta after visiting Paris, Greece, Italy, Czech Republic, and Canada. The individual unknowingly was infected with MDR-TB and, after twelve days of travel, the individual was involuntarily isolated by the CDC in Atlanta under the Public Health Service Act [9]. The CDC held the individual in isolation for one week and then moved him to a hospital in Denver, Colorado [9]. In this case, there are no reported infections resulting from the travel of the individual, perhaps due to the slightly lower infection rate of MDR-TB as compared to drug sensitive TB strains [9]. It should be noted here that the isolation of this individual, albeit brief, potentially helped prevent a rise in drug-resistant TB in the United States, which is currently at admittedly low levels [10]. An occurrence such as this one clearly demonstrates the need for carefully executed isolation procedures for individuals with drug-resistant $\mathrm{TB}$ strains.

Several reports have shown the effectiveness of isolation in reducing the number of people with $\mathrm{TB}[6,7,26$, 27]. Weis et al. [27] showed the effectiveness of isolation, reporting lower occurrences of primary and acquired drug resistance among individuals with TB. Historically, to treat the infection, individuals were isolated in a sanatorium where they would receive proper nutrition and a constant supply of fresh air [28]. However, while this method is successful in certain situations, this treatment methodology is difficult to implement without proper infrastructure in place [29]. Locations without proper facilities such as South Africa have poor treatment and success rates [29]; effective isolation is essential in areas such as these which have high treatment failure rates. Sutton et al. [26] studied three hospitals in California; they found that implementing the CDC isolation guidelines for hospitals was feasible; however, since not every hospital could afford the necessary equipment, the isolation was not the same for each hospital. Even though the results varied, it is noticeably more efficient to isolate patients who are infected with TB.

According to the National Committee of Fight against Tuberculosis of Cameroon [30], about 10\% of infectious individuals who start the recommended WHO DOTS treatment therapy in the hospital do not return to the hospital for the rest of sputum examinations and check-up and are thus lost to follow-up. This can be attributed to the long duration of treatment regimen, negligence, or lack of information about $\mathrm{TB}$ [30], a brief relief from the long term treatment [22], poverty, and so forth. As such, health-care personnel do not know their epidemiological status, that is, if they died, recovered, or are still infectious and this lack of epidemiological status of these individuals can affect the spread of TB in a population [30]. A number of mathematical models for tuberculosis developed account for this population by the inclusion of either the lost sight class [23, 30-32] or lost to follow-up class [22].

The aim of this study is to develop a new deterministic transmission model for TB to gain qualitative insight into the effects of isolation in the presence of individuals who are lost to follow-up on TB transmission dynamics. A notable feature of the model is the incorporation of isolated and lost to follow-up classes for the three TB strains. The paper is organized as follows. The model formulation and analysis is given in Section 2. Sensitivity analysis of the model is considered in Section 3. Analysis of the reproduction number is carried out in Section 4. The effect of isolation is numerically investigated in Section 5. The key theoretical and epidemiological results from this study are summarized in Section 6.

\section{Model Formulation}

The model is formulated as follows: the population is divided into susceptible $(S)$, latently infected $\left(E_{i}\right)$, symptomatically infectious with drug sensitive strain (T), MDR strain $(M)$, XDR strain $(X)$, symptomatically infectious individuals who are lost to follow-up $\left(L_{i}\right)$, isolated $(J)$, and recovered $(R)$, where $i=T, M, X$. Thus, the total population is $N(t)=$ $S(t)+E_{T}(t)+T(t)+E_{M}(t)+M(t)+E_{X}(t)+X(t)+$ $L_{T}(t)+L_{M}(t)+L_{X}(t)+J(t)+R(t)$.

As the disease evolves individuals move from one class to the other with respect to their disease status. The population of susceptible $(S)$ is generated by new recruits (either via birth or immigration) who enter the population at a rate $\pi$. The parameter $\pi$ denotes the recruitment rate. It is assumed that there is no vertical transmission or immigration of infectious; thus, these new inflow does not enter the infectious classes. All individuals, whatever their status, are subject to natural death, which occurs at a rate $\mu$. The susceptible population is reduced by infection following effective contact with infected individuals with drug sensitive, MDR-, and XDR-TB strains at the rates $\lambda_{T}, \lambda_{M}$, and $\lambda_{X}$, where

$$
\begin{aligned}
& \lambda_{T}=\frac{\beta_{T}\left(T+\eta_{T} L_{T}\right)}{N}, \\
& \lambda_{M}=\frac{\beta_{M}\left(M+\eta_{M} L_{M}\right)}{N}, \\
& \lambda_{X}=\frac{\beta_{X}\left(X+\eta_{X} L_{X}\right)}{N} .
\end{aligned}
$$

The parameters $\beta_{T}, \beta_{M}$, and $\beta_{X}$ are the effective transmission probability per contact; we assume that $\beta_{X}<\beta_{M}<\beta_{T}$ [9] and the parameters $\eta_{T}>1, \eta_{M}>1$, and $\eta_{X}>1$ are the modification parameter that indicates the increased infectivity of individuals who are lost to follow-up.

A fraction $l_{T 1}$ of the newly infected individuals with drug sensitive strain move into the latently infected class $\left(E_{T}\right)$, with $l_{T 2}$ fraction moving into the symptomatic-infectious class $(T)$ and the other fraction $\left[1-\left(l_{T 1}+l_{T 2}\right)\right]$ moving into the lost to follow-up class $\left(L_{T}\right)$ with $l_{T 1}+l_{T 2}<1$. The latently infected individuals become actively infectious as a result of endogenous reactivation of the latent bacilli at the rate $\sigma_{T}$.

Similarly a fraction $l_{M 1}$ of the newly infected individuals with MDR stain move into the latently infected class with 
$\operatorname{MDR}\left(E_{M}\right)$, with $l_{M 2}$ fractions moving into the symptomatically infectious class $(M)$ and the other fraction $\left[1-\left(l_{M 1}+\right.\right.$ $\left.l_{M 2}\right)$ ] moving into the symptomatically infectious lost to follow-up class $\left(L_{M}\right)$ with $l_{M 1}+l_{M 2}<1$. The latently infected individuals with MDR strain become actively infectious as a result of endogenous reactivation of the latent bacilli at the rate $\sigma_{M}$.

Lastly, a fraction $l_{X 1}$ of the newly infected individuals with XDR stain moves into the latently infected class with XDR $\left(E_{X}\right)$, with $l_{X 2}$ fractions moving into the symptomatically infectious class $(X)$ and the other fraction $\left[1-\left(l_{X 1}+l_{X 2}\right)\right]$ moving into the symptomatically infectious lost to follow-up class $\left(L_{X}\right)$ with $l_{X 1}+l_{X 2}<1$. The latently infected individuals with XDR strain become actively infectious as a result of endogenous reactivation at the rate $\sigma_{X}$.

Members of the symptomatically infectious class with the drug sensitive strain $(T)$ are lost to follow-up at the rate $\phi_{T}$ and move to the class of lost to follow-up with drug sensitive strain $\left(L_{T}\right)$. They are isolated into the isolated class $(J)$ at the rate $\alpha_{T}$. They undergo the WHO recommended DOTS treatment (Directly Observed Treatment, Short Course (DOTS)); however due to treatment failure (from treatment noncompliance) they move into the latently infected class at the rate $p_{T 1} \gamma_{T}$ or the latently infected class with MDR at the rate $p_{T 2} \gamma_{T}$. The remaining fraction move into the recovered class $(R)$ following effective treatment at the rate $\left(1-p_{T 1}-p_{T 2}\right) \gamma_{T}\left(\right.$ where $\left.p_{T 1}+p_{T 2}<1\right)$. Or they can die from the infection at the rate $\delta_{T}$.

Similarly members of the symptomatically infectious with MDR strain $(M)$ are lost to follow-up at the rate $\phi_{M}$ and move to the class of lost to follow-up with MDR strain $\left(L_{M}\right)$. They are isolated at the rate $\alpha_{M}$. And a fraction of them move into the population of the latently infected with XDR strain as a result of treatment failure of the symptomatically infectious individuals with MDR strain at the rate $p_{M 1} \gamma_{M}$. The remaining fraction move into the recovered class at the rate $\left(1-p_{M 1}\right) \gamma_{M}$ (where $\left.p_{M 1}<1\right)$. Or they can die from the infection at the rate $\delta_{M}$.

Lastly, members of the symptomatically infectious with XDR strain $(X)$ are lost to follow-up at the rate $\phi_{X}$ and move to the class of lost to follow-up with XDR strain $\left(L_{X}\right)$. Or they move into recovered class at the rate $\gamma_{X}$. Or they can die from the infection at the rate $\delta_{X}$. We assume that $\gamma_{X}<\gamma_{M}<\gamma_{T}$.

The individuals who are lost to follow-up $\left(L_{T}\right)$ with drug sensitive strain return at the rate $\psi_{T}$ and move into the class of individuals with drug sensitive strain. Or they die at the rate $\delta_{L T}$. Similarly, the individuals who are lost to follow-up $\left(L_{M}\right)$ with MDR strain return at the rate $\psi_{M}$ and move into the class of individuals with MDR strain. Or they die at the rate $\delta_{L M}$. Lastly, the individuals who are lost to follow-up $\left(L_{X}\right)$ with XDR strain return at the rate $\psi_{X}$ and move into the class of individuals with XDR strain. Or they die at the rate $\delta_{L X}$.

Recovered individuals $(R)$ are reinfected with drug sensitive strain at the rate $\varepsilon \lambda_{T}$, with $l_{T 1} \varepsilon \lambda_{T}$ fraction moving into the latently infected class with drug sensitive strain, $l_{T 2} \varepsilon \lambda_{T}$ fraction moving into the symptomatically infectious class with drug sensitive strain, and the other $\left[1-\left(l_{T 1}+\right.\right.$ $\left.\left.l_{M 2}\right)\right] \varepsilon \lambda_{T}$ moving into the symptomatically infectious lost to follow-up class with drug sensitive strain. Also, these individuals experience reinfection with $\mathrm{MDR}$ and XDR strains and fractions of these move into the latently infected and symptomatically infectious classes, respectively.

It follows, from the above descriptions and assumptions, that the model for the transmission dynamics of the tuberculosis with isolation and lost to follow-up is given by the following deterministic system of nonlinear differential equations (the variables and parameters of the model are described in Table 1; a schematic diagram of the model is depicted in Figure 1):

$$
\begin{aligned}
& \frac{d S}{d t}=\pi-\left[\frac{\beta_{T}\left(T+\eta_{T} L_{T}\right)}{N}+\frac{\beta_{M}\left(M+\eta_{M} L_{M}\right)}{N}\right. \\
& \left.+\frac{\beta_{X}\left(X+\eta_{X} L_{X}\right)}{N}\right] S-\mu S, \\
& \frac{d E_{T}}{d t}=\frac{l_{T 1} \beta_{T}\left(T+\eta_{T} L_{T}\right)(S+\varepsilon R)}{N}+p_{T 1} \gamma_{T} T-\left(\sigma_{T}\right. \\
& +\mu) E_{T} \text {, } \\
& \frac{d T}{d t}=\frac{l_{T 2} \beta_{T}\left(T+\eta_{T} L_{T}\right)(S+\varepsilon R)}{N}+\sigma_{T} E_{T}+\psi_{T} L_{T} \\
& -\left(\phi_{T}+\alpha_{T}+\gamma_{T}+\mu+\delta_{T}\right) T, \\
& \frac{d E_{M}}{d t}=\frac{l_{M 1} \beta_{M}\left(M+\eta_{M} L_{M}\right)(S+\varepsilon R)}{N}+p_{T 2} \gamma_{T} T-\left(\sigma_{M}\right. \\
& +\mu) E_{M} \\
& \frac{d M}{d t}=\frac{l_{M 2} \beta_{M}\left(M+\eta_{M} L_{M}\right)(S+\varepsilon R)}{N}+\sigma_{M} E_{M} \\
& +\psi_{M} L_{M}-\left(\phi_{M}+\alpha_{M}+\gamma_{M}+\mu+\delta_{M}\right) M, \\
& \frac{d E_{X}}{d t}=\frac{l_{X 1} \beta_{X}\left(X+\eta_{X} L_{X}\right)(S+\varepsilon R)}{N}+p_{M 1} \gamma_{M} M-\left(\sigma_{X}\right. \\
& +\mu) E_{X} \text {, } \\
& \frac{d X}{d t}=\frac{l_{X 2} \beta_{X}\left(X+\eta_{X} L_{X}\right)(S+\varepsilon R)}{N}+\sigma_{X} E_{X}+\psi_{X} L_{X} \\
& -\left(\phi_{X}+\alpha_{X}+\gamma_{X}+\mu+\delta_{X}\right) X, \\
& \frac{d L_{T}}{d t}=\frac{\left(1-l_{T 1}-l_{T 2}\right) \beta_{T}\left(T+\eta_{T} L_{T}\right)(S+\varepsilon R)}{N}+\phi_{T} T \\
& -\left(\psi_{T}+\mu+\delta_{L T}\right) L_{T}, \\
& \frac{d L_{M}}{d t}=\frac{\left(1-l_{M 1}-l_{M 2}\right) \beta_{M}\left(M+\eta_{M} L_{M}\right)(S+\varepsilon R)}{N} \\
& +\phi_{M} M-\left(\psi_{M}+\mu+\delta_{L M}\right) L_{M}, \\
& \frac{d L_{X}}{d t}=\frac{\left(1-l_{X 1}-l_{X 2}\right) \beta_{X}\left(X+\eta_{X} L_{X}\right)(S+\varepsilon R)}{N}+\phi_{X} X \\
& -\left(\psi_{X}+\mu+\delta_{L X}\right) L_{X}, \\
& \frac{d J}{d t}=\alpha_{T} T+\alpha_{M} M+\alpha_{X} X-\left(\gamma_{J}+\mu+\delta_{J}\right) J, \\
& \frac{d R}{d t}=\left(1-p_{T 1}-p_{T 2}\right) \gamma_{T} T+\left(1-p_{M 1}\right) \gamma_{M} M+\gamma_{X} X \\
& +\gamma_{J} J-\mu R-\varepsilon\left[\frac{\beta_{T}\left(T+\eta_{T} L_{T}\right)}{N}+\frac{\beta_{M}\left(M+\eta_{M} L_{M}\right)}{N}\right. \\
& \left.+\frac{\beta_{X}\left(X+\eta_{X} L_{X}\right)}{N}\right] R \text {. }
\end{aligned}
$$


TABle 1: Variables and parameters description of model (2).

\begin{tabular}{|c|c|}
\hline Variable & Description \\
\hline$S(t)$ & Susceptible individuals \\
\hline$E_{T}(t), E_{M}(t), E_{X}(t)$ & Latently infected individuals with drug sensitive, MDR, and XDR strains \\
\hline$T(t), M(t), X(t)$ & Symptomatically infectious individuals with drug sensitive, MDR, and XDR strains \\
\hline$L_{T}(t), L_{M}(t), L_{X}(t)$ & Symptomatically infectious individuals who are lost to sight with drug sensitive, MDR, and XDR strains \\
\hline$J(t)$ & Isolated individuals \\
\hline$R(t)$ & Recovered individuals \\
\hline Parameter & Description \\
\hline$\pi$ & Recruitment rate \\
\hline$\mu$ & Natural death rate \\
\hline$\varepsilon$ & $\mathrm{TB}$ reinfection rate \\
\hline$\beta_{T}, \beta_{M}, \beta_{X}$ & Transmission probability \\
\hline$\eta_{T}, \eta_{M}, \eta_{X}$ & Infectivity modification parameter \\
\hline$l_{T 1}, l_{T 2}, l_{M 1}, l_{M 2}, l_{X 1}, l_{X 2}$ & Fraction of fast disease progression \\
\hline$p_{T 1}, p_{T 2}, p_{M 1}$ & Fraction that failed treatment \\
\hline$\sigma_{T}, \sigma_{M}, \sigma_{X}$ & Disease progression rate \\
\hline$\gamma_{T}, \gamma_{M}, \gamma_{X}, \gamma_{J}$ & Recovery rate \\
\hline$\alpha_{T}, \alpha_{M}, \alpha_{X}$ & Isolation rate \\
\hline$\delta_{T}, \delta_{M}, \delta_{X}, \delta_{J}$ & Disease-induced death rate \\
\hline$\phi_{T}, \phi_{M}, \phi_{X}$ & Lost to follow-up rate \\
\hline$\psi_{T}, \psi_{M}, \psi_{X}$ & Return rate from lost to follow-up \\
\hline$\delta_{L T}, \delta_{L M}, \delta_{L X}$ & Disease-induced death rate in individuals who are lost to follow-up \\
\hline
\end{tabular}

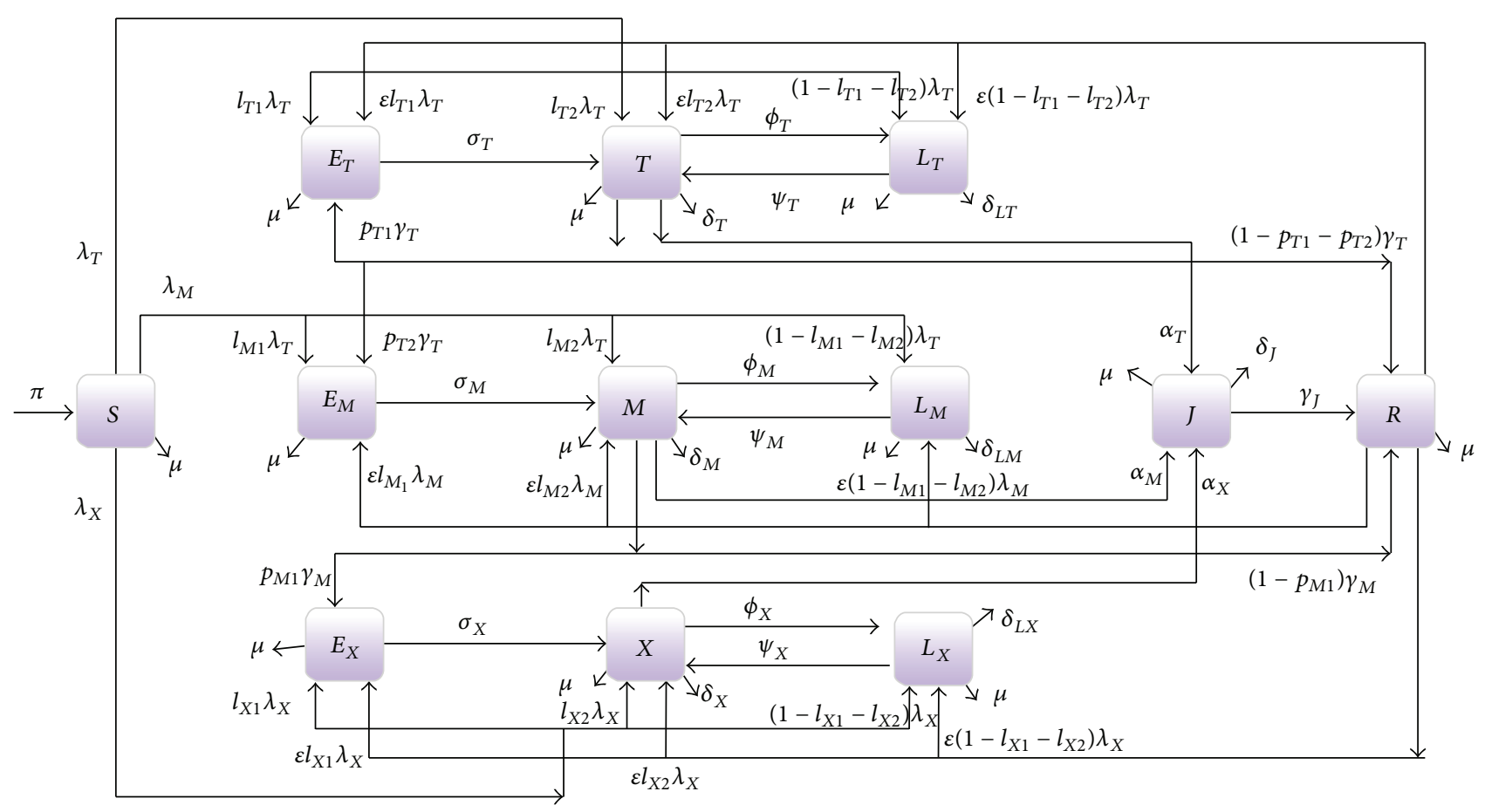

FIgURE 1: Systematic flow diagram of the tuberculosis model (2).

\subsection{Basic Properties}

2.1.1. Positivity and Boundedness of Solutions. For TB model (2) to be epidemiologically meaningful, it can be shown (using the method in Appendix A of [33]) that all its state variables are nonnegative for all time. In other words, solutions of the model system (2) with nonnegative initial data will remain nonnegative for all time $t>0$.

Lemma 1. Let the initial data $S(0) \geq 0, E_{T}(0) \geq 0, T(0) \geq$ $0, E_{M}(0) \geq 0, M(0) \geq 0, E_{X}(0) \geq 0, X(0) \geq 0, L_{T}(0) \geq$ $0, L_{M}(0) \geq 0, L_{X}(0) \geq 0, J(0) \geq 0, R(0) \geq 0$. 
Then the solutions $\left(S, E_{T}, T, E_{M}, M, E_{X}, X, L_{T}, L_{M}, L_{X}, J, R\right)$ of the tuberculosis model (2) are nonnegative for all $t>0$. Furthermore,

$$
\limsup _{t \rightarrow \infty} N(t) \leq \frac{\pi}{\mu}
$$

with

$$
\begin{aligned}
N= & S+E_{T}+T+E_{M}+M+E_{X}+X+L_{T}+L_{M} \\
& +L_{X}+J+R .
\end{aligned}
$$

2.1.2. Invariant Regions. Since model (2) monitors human populations, all variables and parameters of the model are nonnegative. Model (2) will be analyzed in a biologically feasible region as follows. Consider the feasible region

$$
\Phi \subset \mathbb{R}_{+}^{12}
$$

with

$$
\begin{aligned}
\Phi & =\left\{\left(S, E_{T}, T, E_{M}, M, E_{X}, X, L_{T}, L_{M}, L_{X}, J, R\right)\right. \\
& \left.\in \mathbb{R}_{+}^{12}: N(t) \leq \frac{\pi}{\mu}\right\} .
\end{aligned}
$$

The following steps are followed to establish the positive invariance of $\Phi$ (i.e., solutions in $\Phi$ remain in $\Phi$ for all $t>0$ ). The rate of change of the population is obtained by adding the equations of model (2) and this gives

$$
\begin{aligned}
\frac{d N(t)}{d t}= & \pi-\mu N(t)-\delta_{T} T(t)-\delta_{M} M(t)-\delta_{X} X(t) \\
& -\delta_{L T} L_{T}(t)-\delta_{L M} L_{M}(t)-\delta_{L X} L_{X}(t) .
\end{aligned}
$$

And it follows that

$$
\frac{d N(t)}{d t} \leq \pi-\mu N(t)
$$

A standard comparison theorem [34] can then be used to show that

$$
N(t) \leq N(0) e^{-\mu t}+\frac{\pi}{\mu}\left(1-e^{-\mu t}\right) .
$$

In particular, $N(t) \leq \pi / \mu$, if $N(0) \leq \pi / \mu$. Thus, region $\Phi$ is positively invariant. Hence, it is sufficient to consider the dynamics of the flow generated by (2) in $\Phi$. In this region, the model can be considered as being epidemiologically and mathematically well-posed [35]. Thus, every solution of model (2) with initial conditions in $\Phi$ remains in $\Phi$ for all $t>0$. Therefore, the $\omega$-limit sets of system (2) are contained in $\Phi$. This result is summarized below.

Lemma 2. The region $\Phi \subset \mathbb{R}_{+}^{12} \times$ is positively invariant for model (2) with nonnegative initial conditions in $\mathbb{R}_{+}^{12}$.

2.2. Stability of the Disease-Free Equilibrium (DFE). Tuberculosis model (2) has a DFE, obtained by setting the right-hand sides of the equations in the model to zero, given by

$$
\begin{aligned}
& \mathscr{E}_{0} \\
& \quad=\left(S^{*}, E_{T}^{*}, T^{*}, E_{M}^{*}, M^{*}, E_{X}^{*}, X^{*}, L_{T}^{*}, L_{M}^{*}, L_{X}^{*}, J^{*}, R^{*}\right) \\
& \quad=\left(\frac{\pi}{\mu}, 0,0,0,0,0,0,0,0,0,0,0\right) .
\end{aligned}
$$

The linear stability of $\mathscr{E}_{0}$ can be established using the next generation operator method on system (2). Using the notations in [36], the matrices $F$ and $V$, for the new infection terms and the remaining transfer terms, are, respectively, given by

$$
F=\left[F_{1} \mid F_{2}\right],
$$

where

$$
F_{1}=\left(\begin{array}{ccccc}
0 & l_{T 1} \beta_{T} & 0 & 0 & 0 \\
0 & l_{T 2} \beta_{T} & 0 & 0 & 0 \\
0 & 0 & 0 & l_{M 1} \beta_{M} & 0 \\
0 & 0 & 0 & l_{M 2} \beta_{M} & 0 \\
0 & 0 & 0 & 0 & 0 \\
0 & 0 & 0 & 0 & 0 \\
0 & \left(1-l_{T 1}-l_{T 2}\right) \beta_{T} & 0 & 0 & 0 \\
0 & 0 & 0 & \left(1-l_{M 1}-l_{M 2}\right) \beta_{M} & 0 \\
0 & 0 & 0 & 0 & 0 \\
0 & 0 & 0 & 0 & 0
\end{array}\right),
$$




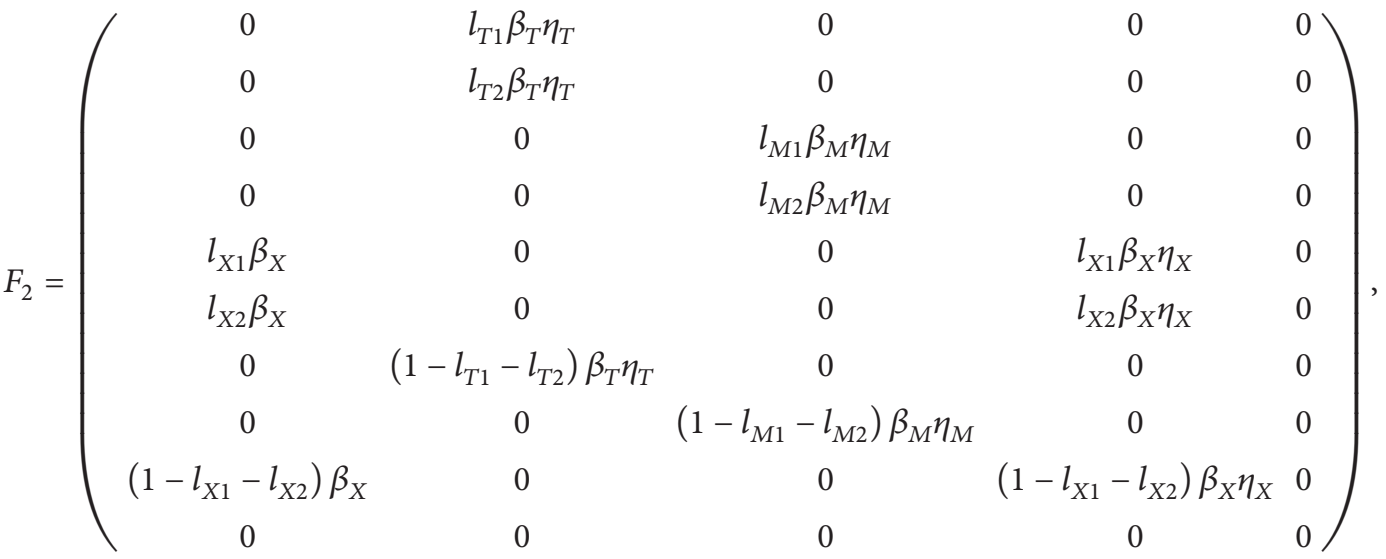

$$
\begin{aligned}
& V=\left(\begin{array}{cccccccccc}
g_{1} & -p_{T 1} \gamma_{T} & 0 & 0 & 0 & 0 & 0 & 0 & 0 & 0 \\
-\sigma_{T} & g_{2} & 0 & 0 & 0 & 0 & -\psi_{T} & 0 & 0 & 0 \\
0 & -p_{T 2} \gamma_{T} & g_{3} & 0 & 0 & 0 & 0 & 0 & 0 & 0 \\
0 & 0 & -\sigma_{M} & g_{4} & 0 & 0 & 0 & -\psi_{M} & 0 & 0 \\
0 & 0 & 0 & -p_{M 1} \gamma_{M} & g_{5} & 0 & 0 & 0 & 0 & 0 \\
0 & 0 & 0 & 0 & -\sigma_{X} & g_{6} & 0 & 0 & -\psi_{X} & 0 \\
0 & -\phi_{T} & 0 & 0 & 0 & 0 & g_{7} & 0 & 0 & 0 \\
0 & 0 & 0 & -\phi_{M} & 0 & 0 & 0 & g_{8} & 0 & 0 \\
0 & 0 & 0 & 0 & 0 & -\phi_{X} & 0 & 0 & g_{9} & 0 \\
0 & -\alpha_{T} & 0 & -\alpha_{M} & 0 & -\alpha_{X} & 0 & 0 & 0 & g_{10}
\end{array}\right),
\end{aligned}
$$

where $g_{1}=\sigma_{T}+\mu, g_{2}=\phi_{T}+\gamma_{T}+\alpha_{T}+\sigma_{T}+\mu, g_{3}=\sigma_{M}+\mu, g_{4}=$ $\phi_{M}+\gamma_{M}+\alpha_{M}+\sigma_{M}+\mu, g_{5}=\sigma_{X}+\mu, g_{6}=\phi_{X}+\gamma_{X}+$ $\alpha_{X}+\sigma_{X}+\mu, g_{7}=\psi_{T}+\mu+\delta_{L T}, g_{8}=\psi_{M}+\mu+\delta_{L M}, g_{9}=$ $\psi_{X}+\mu+\delta_{L X}, g_{10}=\gamma_{J}+\mu+\delta_{J}$.
It follows that the basic reproduction number of tuberculosis model (2), denoted by $\mathscr{R}_{0}$, is given by

$$
\mathscr{R}_{0}=\rho\left(F V^{-1}\right)=\max \left(\mathscr{R}_{T}, \mathscr{R}_{M}, \mathscr{R}_{X}\right),
$$

where

$$
\begin{aligned}
\mathscr{R}_{T} & =\frac{\beta_{T}\left\{\sigma_{T}\left(g_{7}+\phi_{T} \eta_{T}\right) l_{T 1}+\left(g_{7}+\eta_{T} \phi_{T}\right) g_{1} l_{T 2}+\left[\left(g_{2} \eta_{T}+\psi_{T}\right) g_{1}-\eta_{T} \sigma_{T} p_{T 1} \gamma_{T}\right]\left(1-l_{T 1}-l_{T 2}\right)\right\}}{\left[g_{1}\left(g_{7} g_{2}-\phi_{T} \psi_{T}\right)-\eta_{T} \sigma_{T} p_{T 1} \gamma_{T}\right]}, \\
\mathscr{R}_{M} & =\frac{\beta_{M}\left[\sigma_{M}\left(g_{8}+\phi_{M} \eta_{M}\right) l_{M 1}+\left(g_{8}+\eta_{M} \phi_{M}\right) g_{3} l_{M 2}+\left(g_{4} \eta_{M}+\psi_{M}\right) g_{3}\left(1-l_{M 1}-l_{M 2}\right)\right]}{g_{3}\left(g_{8} g_{4}-\phi_{M} \psi_{M}\right)}, \\
\mathscr{R}_{X} & =\frac{\beta_{X}\left[\sigma_{X}\left(g_{9}+\phi_{X} \eta_{X}\right) l_{X 1}+\left(g_{9}+\eta_{X} \phi_{X}\right) g_{5} l_{X 2}+\left(g_{6} \eta_{X}+\psi_{X}\right) g_{5}\left(1-l_{X 1}-l_{X 2}\right)\right]}{g_{5}\left(g_{9} g_{6}-\phi_{X} \psi_{X}\right)} .
\end{aligned}
$$

Quantity $\mathscr{R}_{T}$ represents the reproduction number of TB drug sensitive-only population. Similarly, quantity $\mathscr{R}_{M}$ is the reproduction number for MDR-only population and the quantity $\mathscr{R}_{X}$ represents the reproduction number for XDRonly TB population.

Further, using Theorem 2 in [36], the following result is established.
Lemma 3. The DFE of the tuberculosis model (2), given by $\mathscr{E}_{0}$, is locally asymptotically stable (LAS) if $\mathscr{R}_{0}<1$, and unstable if $\mathscr{R}_{0}>1$.

The threshold quantity $\left(\mathscr{R}_{0}\right.$, i.e., the basic reproduction number) measures the average number of new infections generated by a single infected individual in a completely 


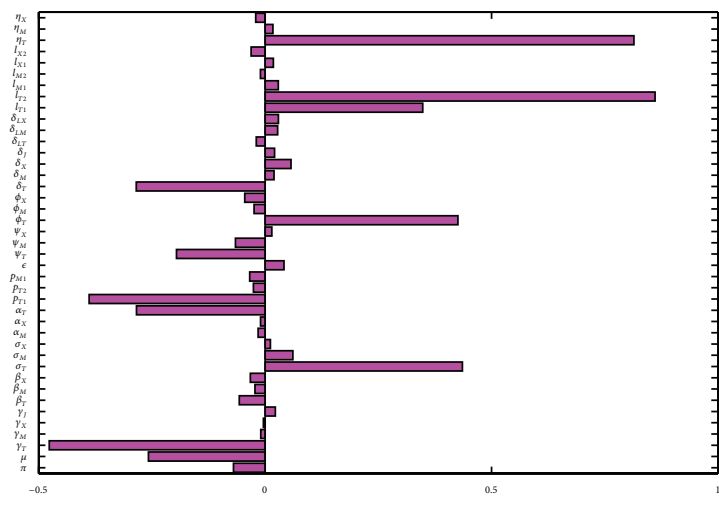

(a)

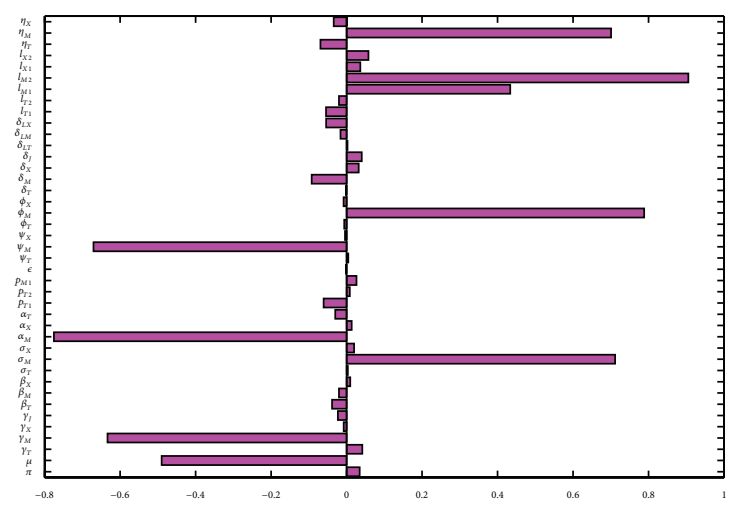

(b)

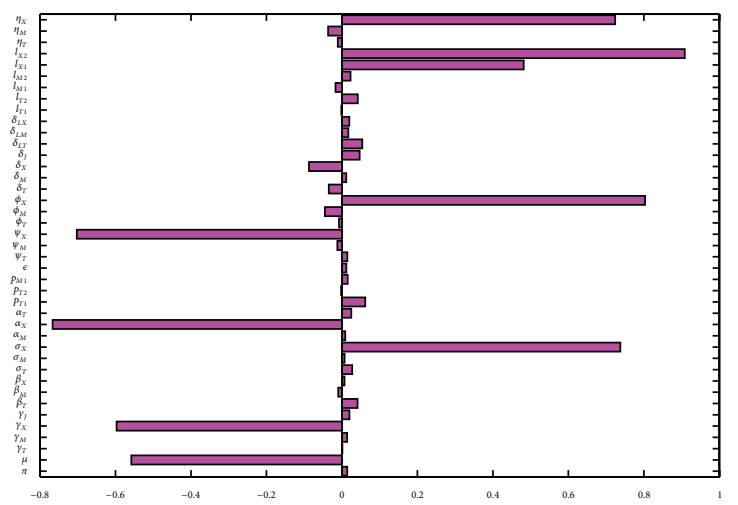

(c)

FiguRE 2: PRCC values for model (2), using as the response function (a) the basic reproduction number ( $\mathscr{R}_{T}$ ), (b) the basic reproduction number $\left(\mathscr{R}_{M}\right)$, and (c) the basic reproduction number $\left(\mathscr{R}_{X}\right)$. Parameter values (baseline) and ranges used are as given in Table 2.

susceptible population [35-38]. Thus, Lemma 3 implies that tuberculosis can be eliminated from the population (when $\left.\mathscr{R}_{0}<1\right)$ if the initial sizes of the subpopulations of model (2) are in the basin of attraction of the DFE, $\mathscr{E}_{0}$.

\section{Sensitivity Analysis}

A global sensitivity analysis [39-42] is carried out, on the parameters of model (2), to determine which of the parameters have the most significant impact on the outcome of the numerical simulations of the model. Figure 2(a) depicts the partial rank correlation coefficient (PRCC) values for each parameter of the models, using the ranges and baseline values tabulated in Table 2 (with the basic reproduction numbers, $\mathscr{R}_{T}$, as the response function), from which it follows that the parameters that have the most influence on drug sensitive TB transmission dynamics are the fraction of fast progression rate $\left(l_{T 2}\right)$ into the drug sensitive TB class, the infectivity modification parameter $\left(\eta_{T}\right)$, the recovery rate $\left(\gamma_{T}\right)$ from drug sensitive TB, disease progression rate $\left(\sigma_{T}\right)$, rate of lost to follow-up $\left(\phi_{T}\right)$ of those with drug sensitive $\mathrm{TB}$, fraction of latently infected with drug sensitive TB that failed treatment $\left(p_{T 1}\right)$, the fraction of fast progression rate $\left(l_{T 1}\right)$ into the latently infected with drug sensitive TB class, and the isolation rate $\left(\alpha_{T}\right)$ from drug sensitive TB class.
The identification of these key parameters is vital to the formulation of effective control strategies for combating the spread of the disease, as this study identifies the most important parameters that drive the transmission mechanism of the disease. In other words, the results of this sensitivity analysis suggest that, to effectively control the spread of drug sensitive $\mathrm{TB}$ in the community, the effective strategy will be to reduce the disease progression rate (reduce $\sigma_{T}$ ), increase the recovery rate (increase $\gamma_{T}$ ) from drug sensitive $\mathrm{TB}$, reduce the disease modification parameter (reduce $\eta_{T}$ ), increase the isolation rate (increase $\alpha_{T}$ ) from drug sensitive TB class, and reduce the fraction of fast progression rates (reduce $l_{T 1}$ and $l_{T 2}$ ) into the drug sensitive TB class and lost to follow-up class and reduce the rate of lost to follow-up (reduce $\phi_{T}$ ) of those with drug sensitive TB. The result of this analysis suggests that the fraction of latently infected with drug sensitive TB that failed treatment $\left(p_{T 1}\right)$ be increased, since this has a negative impact of the basic reproduction number, $\mathscr{R}_{T}$. This of course is counter intuitive; however increasing this rate lowers the rate of development of drug-resistant TB due to treatment failure.

The sensitivity analysis was also carried out using model (2) with the basic reproduction number, $\mathscr{R}_{M}$, for drugresistant $\mathrm{TB}$, as the response function (see Figure 2(b)). The dominant parameters in this case are $\alpha_{M}, \sigma_{M}, \eta_{M}, \gamma_{M}, \phi_{M}$, $\psi_{M}, \mu, l_{M 1}$, and $l_{M 2}$. Similarly, when using as response function the basic reproduction number, $\mathscr{R}_{X}$, for extended drug 
resistance TB (see Figure 2(c)), the dominant parameters in this case are $\alpha_{X}, \sigma_{X}, \eta_{X}, \gamma_{X}, \phi_{X}, \psi_{X}, \mu, l_{X 1}$, and $l_{X 2}$.

The results from these analyses using as response functions the basic reproduction numbers, $\mathscr{R}_{M}$ and $\mathscr{R}_{X}$, suggest that the natural death rate $(\mu)$ be increased, since it has a negative impact on the basic reproduction numbers, $\mathscr{R}_{M}$ and $\mathscr{R}_{X}$. However increasing this rate is not epidemiologically relevant, as it implies reducing the population size that we wish to preserve by other means aside from death by tuberculosis and should therefore be ignored in any control measures.

\section{Analysis of the Reproduction Number}

Following the result obtained in Section 3, we investigate in this section whether or not treatment-only, isolation-only of individuals with tuberculosis or a combination of both can lead to tuberculosis elimination in the population. The analysis will be carried out using the reproduction number for drug sensitive TB $\left(\mathscr{R}_{T}\right)$ since $\mathscr{R}_{0}$ is the maximum of the reproduction number of drug sensitive TB $\left(\mathscr{R}_{T}\right)$, MDR-TB $\left(\mathscr{R}_{M}\right)$, and XDR-TB $\left(\mathscr{R}_{X}\right)$; similar result can be obtained for the MDR- and XDR-TB.

In the absence of isolation $\left(\alpha_{T}=0\right)$, the reproduction number $\left(\mathscr{R}_{T}\right)$ reduces to

$$
\begin{aligned}
& \mathscr{R}_{T_{\alpha}} \\
& \qquad=\frac{\beta_{T}\left[\left(\sigma_{T} g_{7}+\eta_{T} \sigma_{T} \phi_{T}\right) l_{T 1}+\left(\eta_{T} g_{1} \phi_{T}+g_{1} g_{7}\right) l_{T 2}+\left(g_{1} \psi_{T}+\eta_{T} g_{1}\left(\phi_{T}+\gamma_{T}+\mu+\delta_{T}\right)-\eta_{T} \sigma_{T} p_{t 1} \gamma_{T}\right)\left(1-l_{T 1}-l_{T 2}\right)\right]}{g_{1}\left(\phi_{T}+\gamma_{T}+\mu+\delta_{T}\right) g_{7}-g_{1} \psi_{T} \phi_{T}-\sigma_{T} p_{T 1} \gamma_{T} g_{7}} .
\end{aligned}
$$

The reproduction number $\left(\mathscr{R}_{T}\right)$ can be written as

$$
\mathscr{R}_{T}=A_{\alpha} \mathscr{R}_{T_{\alpha}} \text {, }
$$

$$
\begin{aligned}
& A_{\alpha} \\
& =\frac{\left(g_{1} g_{7}\left(\phi_{T}+\gamma_{T}+\mu+\delta_{T}\right)-g_{1} \psi_{T} \phi_{T}-\sigma_{T} p_{T 1} \gamma_{T} g_{7}\right)\left[\left(\sigma_{T} g_{7}+\eta_{T} \sigma_{T} \phi_{T}\right) l_{T 1}+\left(\eta_{T} g_{1} \phi_{T}+g_{1} g_{7}\right) l_{T 2}+\left(g_{1} \psi_{T}+\eta_{T} g_{1} g_{2}-\eta_{T} \sigma_{T} p_{T 1} \gamma_{T}\right)\left(1-l_{T 1}-l_{T 2}\right)\right]}{\left\{\left(g_{1} g_{2} g_{7}-g_{1} \psi_{T} \phi_{T}-\sigma_{T} p_{T 1} \gamma_{T} g_{7}\right)\left[\sigma_{T}\left(g_{7}+\eta_{T} \phi_{T}\right) l_{T 1}+\left(\eta_{T} \phi_{T}+g_{7}\right) g_{1} l_{T 2}+\left(\eta_{T} g_{1}\left(\phi_{T}+\gamma_{T}+\mu+\delta_{T}\right)+g_{1} \psi_{T}-\eta_{T} \sigma_{T} p_{T 1} \gamma_{T}\right)\left(1-l_{T 1}-l_{T 2}\right)\right]\right\}} .
\end{aligned}
$$

The difference between $\mathscr{R}_{T}$ and $\mathscr{R}_{T_{\alpha}}$ is in the isolation rate $\left(\alpha_{T}\right)$; as such the factor $A_{\alpha}$ compares a population with and without isolation; however, this is in the presence of treatment and individuals who are lost to follow-up and individuals returning from lost to follow-up. If $\mathscr{R}_{T_{\alpha}}<1$, then drug sensitive TB cannot develop into an epidemic in the community. However, if $\mathscr{R}_{T_{\alpha}}>1$, it is imperative to investigate the effect of isolation on the transmission of drug sensitive TB among the populace and determine the necessary condition for slowing down its development in the community. Following [43] we have

$$
\Delta_{\alpha}=\mathscr{R}_{T_{\alpha}}-\mathscr{R}_{T}=\left(1-A_{\alpha}\right) \mathscr{R}_{T_{\alpha}},
$$

where

$$
\Delta_{\alpha}=\frac{\left[\beta_{T} g_{1}\left(g_{7}+\eta_{T} \phi_{T}\right)\left(\phi_{T}-g_{2}+\gamma_{T}+\mu+\delta_{T}\right)\left(\sigma_{T} g_{7} l_{T 1}+g_{1} g_{7} l_{T 2}+g_{1} \psi_{T}\left(1-l_{T 1}-l_{T 2}\right)\right)\right]}{\left\{\left(g_{1} g_{2} g_{7}-g_{1} \psi_{T} \phi_{T}-\sigma_{T} p_{T 1} \gamma_{T} g_{7}\right)\left[g_{1} g_{7}\left(\phi_{T}+\gamma_{T}+\mu+\delta_{T}\right)-g_{1} \psi_{T} \phi_{T}-\sigma_{T} p_{T 1} \gamma_{T} g_{7}\right]\right\}}
$$

To slow down the spread of drug sensitive tuberculosis in the population via effective isolation, proper treatment, and identification of individuals who return from lost to followup and in the presence of individuals who are lost to followup, we expect that $\Delta_{\alpha}>0$, and this is satisfied if $A_{\alpha}<1$ in (18). Now, setting $\mathscr{R}_{T}=1$ and solving for $A_{\alpha}$ gives the threshold effectiveness of isolation and taking into account treatment and identification of individuals who return from lost to follow-up and who are also lost to follow-up:

$$
A_{\alpha}^{*}=\frac{1}{\mathscr{R}_{T_{\alpha}}} \text {. }
$$

Hence, drug sensitive tuberculosis can be eradicated in the community in the presence of isolation taking into 
consideration proper treatment and identification of individuals who return from lost to follow-up and are lost to followup if $A_{\alpha}<A_{\alpha}^{*}$ is attained. Note that $A_{\alpha}^{*}$ is a decreasing function of $\mathscr{R}_{T_{\alpha}}$, thus indicating that higher values for $A_{\alpha}^{*}$ will result in smaller values for $\mathscr{R}_{T_{\alpha}}$, a desired outcome. But a large value for $\mathscr{R}_{T_{\alpha}}$ results in a small value for $A_{\alpha}^{*}$, an indication that eradication may not be attainable.

The following limits of $A_{\alpha}$ provide a further insight into possible ways of reducing the burden of drug sensitive TB in the community:

$$
\begin{aligned}
& \lim A_{\alpha \alpha_{T} \rightarrow \infty} \\
& \quad=\frac{l_{T 2} \eta_{T}\left[\left(\phi_{T}+\mu+\delta_{T}+\gamma_{T}\right) g_{1} g_{7}-\psi_{T} \phi_{T} g_{1}-\sigma_{T} p_{T 1} \gamma_{T} g_{7}\right]}{g_{7}\left\{\sigma_{T}\left(g_{7}+\eta_{T} \phi_{T}\right) l_{T 1}+\left(\eta_{T} \phi_{T}+g_{7}\right) g_{1} l_{T 2}+\left[\psi_{T} g_{1}+\eta_{T}\left(\phi_{T}+\gamma_{T}+\mu+\delta_{T}\right) g_{1}-\eta_{T} \sigma_{T} p_{T 1} \gamma_{T}\right]\left(1-l_{T 1}-l_{T 2}\right)\right\}} \\
& \lim A_{\alpha \phi_{T} \rightarrow \infty}=1, \\
& \lim A_{\alpha \psi_{T} \rightarrow \infty}=1, \\
& \lim A_{\alpha \gamma_{T} \rightarrow \infty}=1 .
\end{aligned}
$$

The limits in (21) will be less than unity. In practice, $\alpha_{T} \rightarrow \infty$ implies high rate of isolating individuals with drug sensitive $\mathrm{TB}, \phi_{\mathrm{T}} \rightarrow \infty$ implies high rate of individuals who are lost to follow-up, $\psi_{T} \rightarrow \infty$ implies a high rate of individuals who return from lost to follow-up, and $\gamma_{T} \rightarrow \infty$ implies high treatment rate. Ideally, the results obtained from these limits can be pursued for the reduction of the burden of drug sensitive TB in the community, provided of course that it is feasible and practicable economically. Therefore, with a look at factor $A_{\alpha}$, one observes that an effective isolation will lead to a reduction in the burden of drug sensitive TB in the population.

Next, we consider the case when the treatment rate is set to zero $\left(\gamma_{T}=0\right)$. The reproduction number is given as

$$
\mathscr{R}_{T_{\gamma}}=\frac{\beta_{T}\left[\left(\sigma_{T} g_{7}+\eta_{T} \sigma_{T} \phi_{T}\right) l_{T 1}+\left(\eta_{T} g_{1} \phi_{T}+g_{1} g_{7}\right) l_{T 2}+\left[g_{1} \psi_{T} \eta_{T} g_{1}\left(\phi_{T}+\alpha_{T}+\mu+\delta_{T}\right)\right]\left(1-l_{T 1}-l_{T 2}\right)\right]}{g_{1}\left(\phi_{T}+\alpha_{T}+\mu+\delta_{T}\right) g_{7}-g_{1} \psi_{T} \phi_{T}} .
$$

The reproduction number $\mathscr{R}_{T}$ can be expressed as

$$
\mathscr{R}_{T}=A_{\gamma} \mathscr{R}_{T_{\gamma}}
$$

$$
\begin{aligned}
& A_{\gamma} \\
& =\frac{\left\{\left[\left(\sigma_{T} g_{7}+\eta_{T} \sigma_{T} \phi_{T}\right) l_{T 1}+\left(\eta_{T} g_{1} \phi_{T}+g_{1} g_{7}\right) l_{T 2}+\left(g_{1} \psi_{T}+\eta_{T} g_{1} g_{2}-\eta_{T} \sigma_{T} p_{T 1} \gamma_{T}\right)\left(1-l_{T 1}-l_{T 2}\right)\right] g_{1}\left[\left(\phi_{T}+\alpha_{T}+\mu+\delta_{T}\right) g_{7}-\psi_{T} \phi_{T}\right]\right\}}{\left\{\left(g_{1} g_{2} g_{7}-g_{1} \psi_{T} \phi_{T}-\sigma_{T} p_{t 1} \gamma_{T} g_{7}\right)\left[\left(\sigma_{T} g_{7}+\eta_{T} \sigma_{T} \phi_{T}\right) l_{T 1}+\left(\eta_{T} g_{1} \phi_{T}+g_{1} g_{7}\right) l_{T 2}+\left(g_{1} \psi_{T}+\left(\phi_{T}+\alpha_{T}+\mu+\delta_{T}\right) g_{1} \eta_{T}\right)\left(1-l_{T 1}-l_{T 2}\right)\right]\right\}}
\end{aligned}
$$

The difference between $\mathscr{R}_{T_{\gamma}}$ and $\mathscr{R}_{T}$ is in the treatment rate $\left(\gamma_{T}\right)$; thus $A_{\gamma}$ compares a population with and without treatment in the presence of isolation of infected individuals with drug sensitive TB, individuals who are lost to followup and who return from of lost to follow-up. If $\mathscr{R}_{T_{\gamma}}<1$, then drug sensitive TB cannot develop into an epidemic in the community and no control strategy will be required for its control. Take the difference between $\mathscr{R}_{T}$ and $\mathscr{R}_{T_{\gamma}}$; that is,

$$
\Delta_{\gamma}=\mathscr{R}_{T}-\mathscr{R}_{T_{\gamma}}=\left(1-A_{\gamma}\right) \mathscr{R}_{T_{\gamma}},
$$

where

$$
\Delta_{\gamma}=\frac{\left\{\beta_{T}\left(g_{7}+\eta_{T} \phi_{T}\right)\left[g_{1}\left(\phi_{T}+\mu+\delta_{T}+\alpha_{T}-g_{2}\right)+\gamma_{T} p_{T 1} \sigma_{T}\right]\left[g_{1} g_{7} l_{T 2}+\sigma_{T} g_{7} l_{T 1}+g_{1} \psi_{T}\left(1-l_{T 1}-l_{T 2}\right)\right]\right\}}{\left\{g_{1}\left(g_{1} g_{2} g_{7}-g_{1} \psi_{T} \phi_{T}-\sigma_{T} p_{T 1} \gamma_{T} g_{7}\right)\left[g_{7}\left(\phi_{T}+\alpha_{T}+\mu+\delta_{T}\right)-\psi_{T} \phi_{T}\right]\right\}}
$$


To slow down the spread of drug sensitive tuberculosis in the population using proper treatment, effective isolation and identification of individuals who return from lost to follow-up and in the presence of those who are of lost to follow-up, we expect that $\Delta_{\gamma}>0$, and this is satisfied if $A_{\gamma}<1$ in (18).

Take the following limits of $A_{\gamma}$ :

$$
\begin{aligned}
& \lim A_{\gamma_{\gamma_{T} \rightarrow \infty}} \\
& =\frac{\left[\left(\phi_{T}+\alpha_{T}+\mu+\delta_{T}\right) g_{7}-\psi_{T} \phi_{T}\right] \eta_{T} g_{1}\left(1-l_{T 1}-l_{T 2}\right)}{g_{7}\left\{\sigma_{T}\left(g_{7}+\eta_{T} \phi_{T}\right) l_{T 1}+\left(\eta_{T} \phi_{T}+g_{7}\right) g_{1} l_{T 2}+\left[\psi_{T} g_{1}+\eta_{T}\left(\phi_{T}+\gamma_{T}+\mu+\delta_{T}\right) g_{1}-\eta_{T} \sigma_{T} p_{T 1} \gamma_{T}\right]\left(1-l_{T 1}-l_{T 2}\right)\right\}}, \\
& \lim A_{\gamma_{\phi_{T} \rightarrow \infty}}=1, \\
& \lim A_{\gamma_{\psi_{T} \rightarrow \infty}}=1, \\
& \lim A_{\gamma_{\alpha_{T} \rightarrow \infty}}=1 .
\end{aligned}
$$

From the limit of $A_{\gamma}$, one observes that an effective treatment will lead to a reduction in the burden of drug sensitive TB in the population.
Comparing the quantities $A_{\alpha}$ and $A_{\gamma}$ shows that $A_{\alpha}<$ $A_{\gamma}$; that is, size

$$
\begin{aligned}
A_{\alpha}-A_{\gamma}=-\left\{[ ( g _ { 7 } + \eta _ { T } \phi _ { T } ) + ( g _ { 7 } + \eta _ { T } \phi _ { T } ) l _ { T 2 } g _ { 1 } + ( \psi _ { T } g _ { 1 } + \eta _ { T } g _ { 1 } g _ { 2 } - \eta _ { T } \sigma _ { T } p _ { T 1 } \gamma _ { T } ) ( 1 - l _ { T 1 } - l _ { T 2 } ) ] \left[g_{1}\left(\alpha_{T}-\gamma_{T}\right)\right.\right. \\
\left.\left.\quad+\sigma_{T} p_{T 1} \gamma_{T}\right]\left(g_{7}+\eta_{T} \phi_{T}\right)\left[\sigma_{T} g_{7} l_{T 1}+g_{1} g_{7} l_{T 2}+g_{1} \psi_{T}\left(1-l_{T 1}-l_{T 2}\right)\right]\right\}\left(\left\{\left(g_{1} g_{2} g_{7}-g_{1} \psi_{T} \phi_{T}-\sigma_{T} p_{T 1} \gamma_{T} g_{7}\right)\right.\right. \\
\cdot\left[\sigma_{T}\left(g_{7} \eta_{T} \phi_{T}\right) l_{T 1}+\left(g_{7}+\eta_{T} \phi_{T}\right) g_{1} l_{T 2}+\left(\psi_{T} g_{1}+\eta_{T} g_{1}\left(\phi_{T}+\alpha_{T}+\mu+\delta_{T}\right)\right)\left(1-l_{T 1}-l_{T 2}\right)\right] \\
\left.\left.\cdot\left[\sigma_{T}\left(g_{7}+\eta_{T} \phi_{T}\right) l_{T 1}+\left(g_{7}+\eta_{T} \phi_{T}\right) g_{1} l_{T 2}+\left(\psi_{T} g_{1}+\eta_{T} g_{1}\left(\phi_{T}+\gamma_{T}+\mu+\delta_{T}\right)-\eta_{T} \sigma_{T} p_{T 1} \gamma_{T}\right)\left(1-l_{T 1}-l_{T 2}\right)\right]\right\}\right)^{-1}<0 .
\end{aligned}
$$

This implies that $A_{\gamma}$ will provide better results in slowing down drug sensitive tuberculosis spread, using reduction in the prevalence, than using $A_{\alpha}$. On the other hand if $A_{\alpha}-A_{\gamma}>$ 0 , this means that $A_{\alpha}$ will give better outcome in slowing down drug sensitive tuberculosis spread compared to using $A_{\gamma}$.

Thus, from the above discussions, to slow down the spread of the disease and reduce the number of secondary infections in the population, we require control strategies with parameter values that would make $A_{\alpha}<1$ or $A_{\gamma}<$ 1. Hence, the necessary condition for slowing down the development of drug sensitive TB at the population level is that $\Delta_{\alpha}>0$ or $\Delta_{\gamma}>0$. However, $\Delta_{\alpha}$ gives a better result in terms of reduction in the prevalence of the disease over $\Delta_{\gamma}$ provided $\Delta_{\gamma}>\Delta_{\alpha}$; otherwise if $\Delta_{\alpha}>\Delta_{\gamma}$, then $\Delta_{\gamma}$ gives a better result over $\Delta_{\alpha}$. Using parameters in Table 1, we have that $A_{\alpha}=0.8524, A_{\gamma}=0.8802$ and $\Delta_{\alpha}=0.1061$, $\Delta_{\gamma}=0.0833$; thus $A_{\alpha}-A_{\gamma}=-0.02789$. It follows that, the isolation-only strategy provides more effective control measures in curtailing the disease transmission in the community.

Using the threshold quantity, $\mathscr{R}_{T}$, we determine how isolation and treatment rates could lead to tuberculosis elimination in the population. Thus

$$
\begin{aligned}
& \lim \mathscr{R}_{T \alpha_{T} \rightarrow \infty}=\frac{\beta_{T}\left(1-l_{T 1}-l_{T 2}\right) \eta_{T}}{\left(\psi_{T}+\mu+\delta_{T}\right)}>0, \\
& \lim \mathscr{R}_{T \gamma_{T} \rightarrow \infty}=\frac{\beta_{T}\left(1-l_{T 1}-l_{T 2}\right) \eta_{T}}{\left(\psi_{T}+\mu+\delta_{T}\right)}>0 .
\end{aligned}
$$

Thus a sufficient effective TB control program that isolates (or treats) the identified cases at a high rate $\alpha_{T} \rightarrow \infty\left(\right.$ or $\gamma_{T} \rightarrow$ $\infty)$ can lead to effective disease control if it results in making the respective right-hand side of (30) less than unity.

Differentiating partially the reproduction number of $\partial \mathscr{R}_{T}$ with respect to the key parameters $\left(\alpha_{T}\right.$ and $\left.\gamma_{T}\right)$, this gives

$$
\begin{aligned}
\frac{\partial \mathscr{R}_{T}}{\partial \alpha_{T}} & =\frac{-\beta_{T} g_{1}\left(g_{7}+\eta_{T} \phi_{T}\right)\left[l_{T 1} \sigma_{T} g_{7}+l_{T 2} g_{1} g_{7}+\left(1-l_{T 1}-l_{T 2}\right) g_{1} \psi_{T}\right]}{\left[g_{1} g_{7}\left(\phi_{T}+\alpha_{T}+\gamma_{T}+\mu+\delta_{T}\right)-g_{1} \psi_{T} \phi_{T}-\sigma_{T} p_{T 1} \gamma_{T} g_{7}\right]^{2}} \\
\frac{\partial \mathscr{R}_{T}}{\partial \gamma_{T}} & =\frac{-\beta_{T}\left(g_{1}-\sigma_{T} p_{t 1}\right)\left(g_{7}+\eta_{T} \phi_{T}\right)\left[l_{T 1} \sigma_{T} g_{7}+l_{T 2} g_{1} g_{7}+\left(1-l_{T 1}-l_{T 2}\right) g_{1} \psi_{T}\right]}{\left[g_{1} g_{7}\left(\phi_{T}+\alpha_{T}+\gamma_{T}+g_{1} g_{7} \mu+\delta_{T}\right)-g_{1} \psi_{T} \phi_{T}-\sigma_{T} p_{T 1} \gamma_{T} g_{7}\right]^{2}}
\end{aligned}
$$


TABLE 2: Values of the parameters of the model (2).

\begin{tabular}{|c|c|c|c|}
\hline Parameter & $\begin{array}{l}\text { Baseline } \\
\text { values }\end{array}$ & Range & Reference \\
\hline$\pi$ & $\mu \times 10^{5}$ & $\begin{array}{c}(0.0143 \times \\
\left.10^{5}, 0.04 \times 10^{5}\right)\end{array}$ & [11] \\
\hline$\mu$ & 0.0159 & $(0.0143,0.04)$ & {$[12]$} \\
\hline$\varepsilon$ & 0.06 & $(0.01,0.5)$ & [13] \\
\hline$\beta_{T}$ & 9.75 & $(4.5,15.0)$ & {$[14]$} \\
\hline$\beta_{M}$ & 1.5 & $(1.5,3.5)$ & {$[13,15]$} \\
\hline$\beta_{X}$ & 0.0000085 & $(0.01,0.1)$ & {$[13]$} \\
\hline$\eta_{T}, \eta_{M}, \eta_{X}$ & 0.5 & $(0,1)$ & Assumed \\
\hline$l_{T 1}, l_{T 2}, l_{M 1}, l_{M 2}, l_{X 1}, l_{X 2}$ & 0.14 & $(0.02,0.3)$ & {$[12,16,17]$} \\
\hline$p_{T 1}$ & 0.3 & $(0.1,0.5)$ & {$[14,18]$} \\
\hline$p_{T 2}$ & 0.03 & $(0.01,0.05)$ & {$[14,18]$} \\
\hline$p_{M 1}$ & 0.03 & $(0.01,0.1)$ & {$[14,18]$} \\
\hline$\sigma_{T}, \sigma_{M}, \sigma_{X}$ & $\begin{array}{c}0.05,0.0018 \\
0.013\end{array}$ & $(0.005,0.05)$ & {$[12,19,20]$} \\
\hline$\gamma_{T}, \gamma_{J}$ & 1.5 & $(1.5,2.5)$ & {$[11,16,21]$} \\
\hline$\gamma_{M}, \gamma_{X}$ & 0.75 & $(0.5,1.0)$ & [13] \\
\hline$\alpha_{T}, \alpha_{M}, \alpha_{X}$ & 0.6 & $(0.2,1.0)$ & Assumed \\
\hline$\phi_{T}, \phi_{M}, \phi_{X}$ & 0.2511 & $(0.0022,0.5)$ & [22] \\
\hline$\psi_{T}, \psi_{M}, \psi_{X}$ & 0.1 & $(0.5,1.0)$ & [23] \\
\hline$\delta_{T}, \delta_{J}$ & 0.365 & $(0.22,0.39)$ & {$[20,24,25]$} \\
\hline$\delta_{M}, \delta_{X}$ & 0.028 & $(0.01,0.03)$ & [13] \\
\hline$\delta_{L T}, \delta_{L M}, \delta_{L X}$ & 0.02 & $(0.01,0.039)$ & {$[22,23]$} \\
\hline
\end{tabular}

Thus, it follows from (31) that $\partial \mathscr{R}_{T} / \partial \alpha_{T}<0$, hence showing further the effectiveness of the control measures. Thus, isolation $\left(\alpha_{T}\right)$ of drug sensitive tuberculosis will have a positive impact in reducing the drug sensitive TB burden in the community, regardless of the values of the other parameters. This result is stated in the following lemma.

Lemma 4. The use of isolation $\left(\alpha_{T}\right)$ will have a positive impact on the reduction of the drug sensitive TB burden in a community regardless of the values of other parameters in the basic reproduction number under isolation.

Similarly, from (32), we have that $\partial \mathscr{R}_{T} / \partial \gamma_{T}<0$. Thus, effective treatment $\left(\gamma_{T}\right)$ of drug sensitive tuberculosis will have a positive impact in reducing the drug sensitive tuberculosis burden in the community, irrespective of the values of the other parameters. This result is summarized below.

Lemma 5. The use of effective treatment $\left(\gamma_{T}\right)$ will have a positive impact on the reduction of the drug sensitive TB burden in a community irrespective of the values of other parameters in the basic reproduction number under treatment.

A contour plot of the reproduction number $\mathscr{R}_{T}$, as a function of the effective treatment rate $\left(\gamma_{T}\right)$ and isolation rate $\left(\alpha_{T}\right)$, is depicted in Figure 3(a). As expected, the plot shows a decrease in $\mathscr{R}_{T}$ values with increasing values of the treatment and isolation rates. For instance, if the use of effective treatment result in $\gamma_{T}=0.8$ and $\alpha_{T}=0.8$, drug sensitive TB burden will be reduced considerably in the population. Similarly in Figure 3(b) the plot shows a decrease in $\mathscr{R}_{T}$ values with decreasing values of the return rate from lost to follow-up $\left(\psi_{T}\right)$ and lost to follow-up rate $\left(\phi_{T}\right)$.

A contour plot of the reproduction number $\mathscr{R}_{T}$, as a function of return rate from lost to follow-up $\left(\psi_{T}\right)$ and the effective treatment rate $\left(\gamma_{T}\right)$, is depicted in Figure 4(a). The plot shows a decrease in $\mathscr{R}_{T}$ values with increasing values of the treatment rate. Similarly the plot in Figure 4(b) shows a decrease in $\mathscr{R}_{T}$ values with increasing values of the isolation rate $\left(\alpha_{T}\right)$.

4.1. Backward Bifurcation Analysis. Model (2) is now investigated for the possibility of the existence of the phenomenon of backward bifurcation (where a stable DFE coexists with a stable endemic equilibrium when the reproduction number, $\mathscr{R}_{0}$, is less than unity) [44-53]. The epidemiological implication of backward bifurcation is that the elimination (or effective control) of the TB (and various strains) in the system is no longer guaranteed when the reproduction number is less than unity but is dependent on the initial sizes of the subpopulations. The possibility of backward bifurcation in model (2) is explored using the centre manifold theory [47], as described in [54] (Theorem 4.1).

Theorem 6. Model (2) undergoes a backward bifurcation at $\mathscr{R}_{T}=1$ whenever inequality (A.9), given in Appendix A, holds.

The proof of Theorem 6 is given in Appendix A (the proof can be similarly given for the case when $\mathscr{R}_{M}=1$ or $\mathscr{R}_{X}=1$ ). The backward bifurcation property of model (2) is illustrated by simulating the model using a set of parameter values given in Table 2 (such that the bifurcation parameters, $a$ and $b$, given in Appendix A, take the values $a=558.61>0$ and $b=$ $1.59>0$, resp.). The backward bifurcation phenomenon of model (2) makes the effective control of the TB strains in the population difficult, since, in this case, disease control when $\mathscr{R}_{0}<1$ is dependent on the initial sizes of the subpopulations of model (2). This phenomenon is illustrated numerically in Figures 5 and 6 for individuals with drug sensitive, MDR-, and XDR-TB, as well as individuals who are lost to follow-up with drug sensitive, MDR-, and XDR-TB, respectively.

It is worth mentioning that when the reinfection parameter of model (2), for the recovered individuals, is set to zero (i.e., $\varepsilon=0$ ), the bifurcation parameter, $a$, becomes negative (see Appendix A). This rules out backward bifurcation (in line with Item (iv) of Theorem 4.1 of [54]) in this case. Thus, this study shows that the reinfection of recovered individuals causes backward bifurcation in the transmission dynamics of TB in the system. To further confirm the absence of the backward bifurcation phenomenon in model (2) for this case, the global asymptotic stability of the DFE of the model is established below for the case when no reinfection of recovered individuals occurs. 


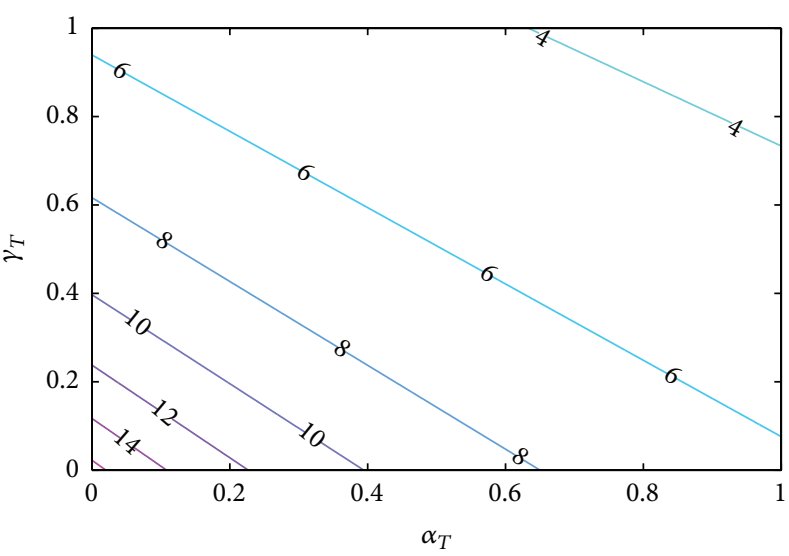

(a)

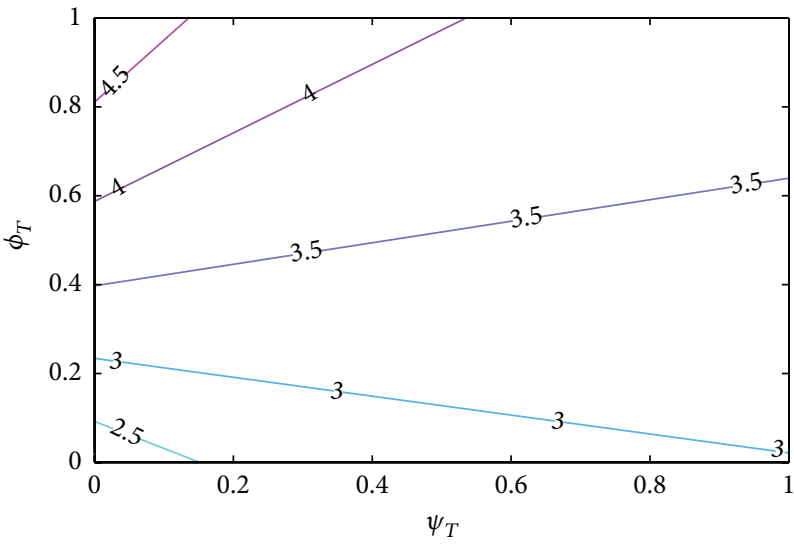

(b)

FIGURE 3: Contour plot of the reproduction number $\left(\mathscr{R}_{T}\right)$ of model (2) as: (a) a function of isolation rate $\left(\alpha_{T}\right)$ and treatment rate $\left(\gamma_{T}\right)$; (b) a function of return rate from lost to follow-up $\left(\psi_{T}\right)$ and lost to follow-up rate $\left(\phi_{T}\right)$. Parameter values used are as given in Table 2.

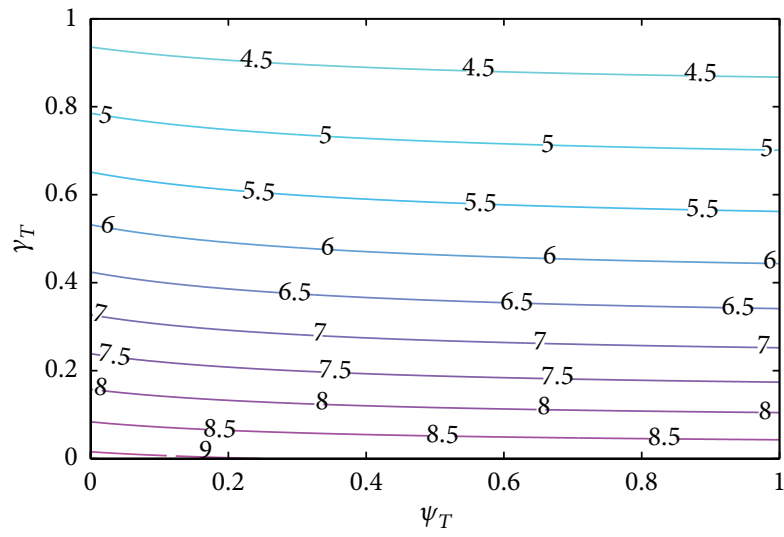

(a)

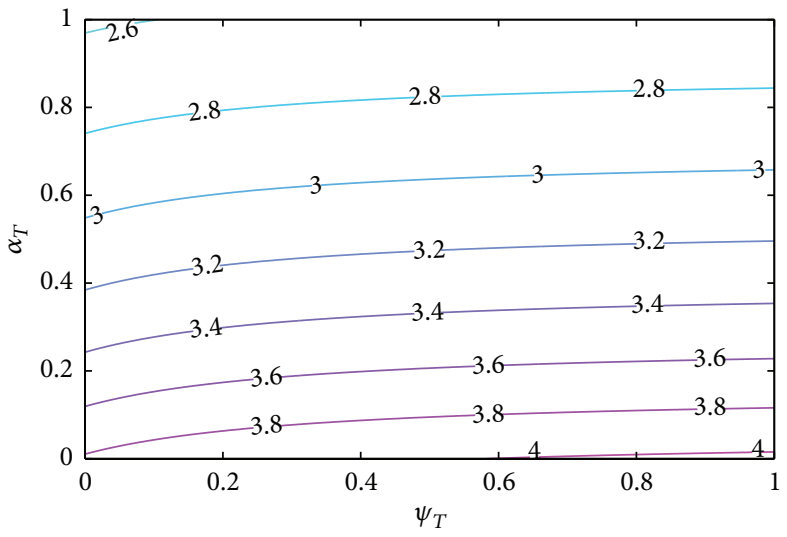

(b)

Figure 4: Contour plot of the reproduction number $\left(\mathscr{R}_{T}\right)$ of model (2) as: (a) a function of return rate from lost to follow-up $\left(\psi_{T}\right)$ and treatment rate $\left(\gamma_{T}\right)$; (b) a function of return rate from lost to follow-up $\left(\psi_{T}\right)$ and treatment rate $\left(\alpha_{T}\right)$. Parameter values used are as given in Table 2.

4.2. Global Stability of the DFE: Special Case. Consider the special case of model (2) where the reinfection parameters are set to zero (i.e., $\varepsilon=0$ ). It is convenient to define the reproduction threshold $\widetilde{\mathscr{R}}_{0}=\left.\mathscr{R}_{0}\right|_{\varepsilon=0}$.

Theorem 7. The DFE of model (2), with $\varepsilon=0$, is GAS in $\Phi$ whenever $\widetilde{\mathscr{R}}_{0}<1$.

The proof of Theorem 7 is given in Appendix B.

The epidemiological significance of Theorem 7 is that, for the special case of model (2) with $\varepsilon=0$, TB will be eliminated from the community if the reproduction number $\left(\widetilde{\mathscr{R}}_{0}\right)$ can be brought to (and maintained at) a value less than unity.

\section{The Effects of Isolation}

Following the result obtained from the sensitivity analysis, we investigate the impact of the isolation parameters $\alpha_{T}, \alpha_{M}$, and
$\alpha_{X}$, which are one of the dominant parameters of model (2). We start by individually varying these parameters for (say) $\alpha_{T}=0.2,0.4,0.6,0.8,1.0$, with the other parameters given in Table 2 kept constant. We observed that (see Figure 7) as the isolation rate, $\alpha_{T}$, for drug sensitive TB increases, the total number of individuals (with drug sensitive, MDR, and XDR) isolated with each strain of TB increases, while the total number of individuals who are lost to follow-up decreases. We observed similar result for the isolation rate $\alpha_{M}$ (see Figure 8). However, for isolation rate $\alpha_{X}$, negligible change was observed and the plots are not shown.

\section{Conclusion}

In this paper, we have developed and analyzed a system of ordinary differential equations for the transmission dynamics of drug-resistant tuberculosis with isolation. From our analysis, we have the following results which are summarized below: 


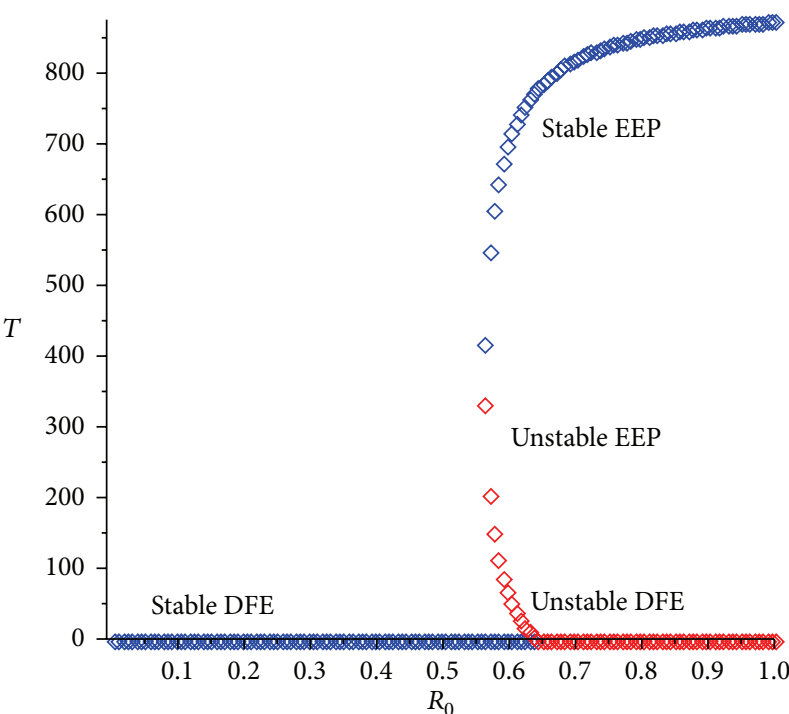

(a)

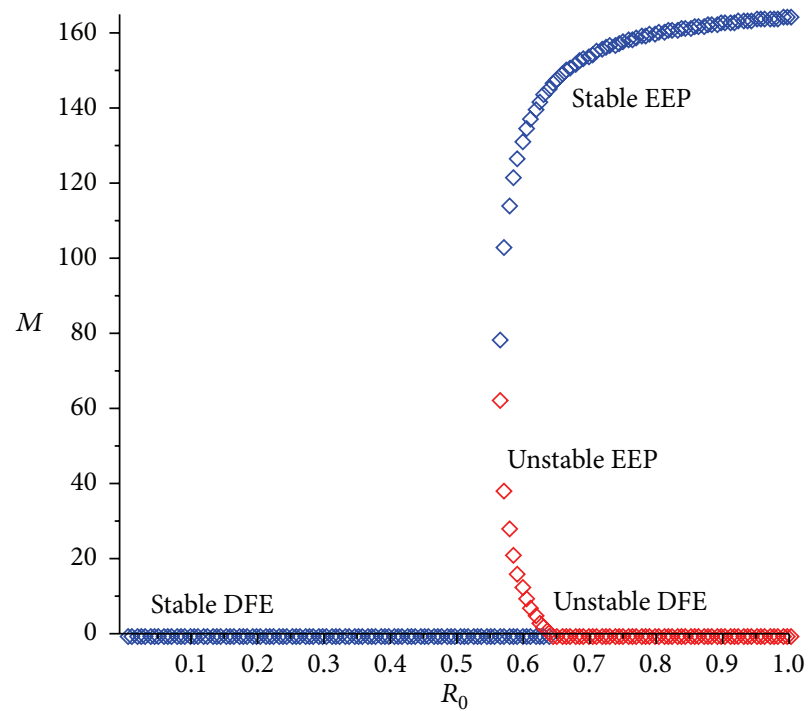

(b)

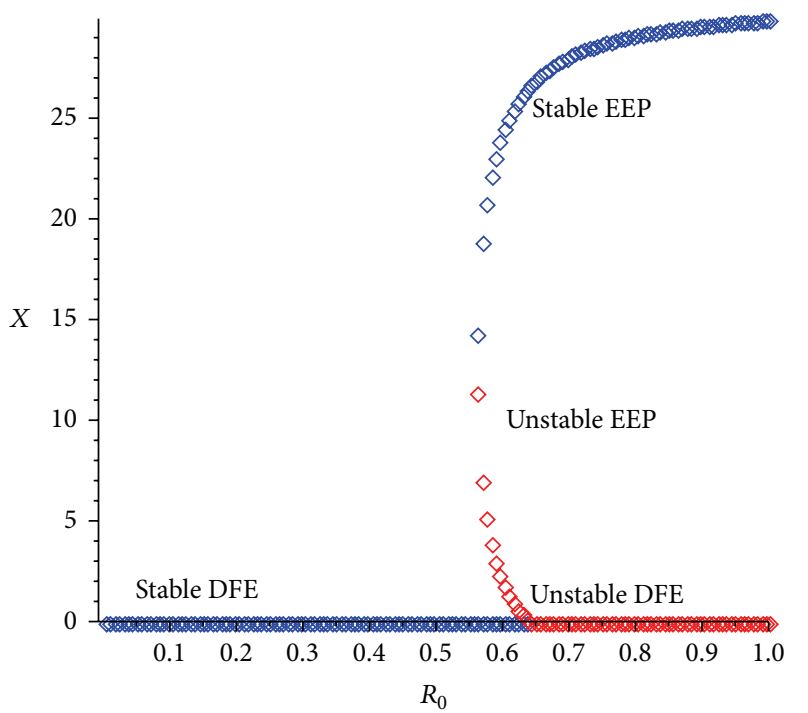

(c)

FIGURE 5: Backward bifurcation plot of model (2) as a function of time. (a) Individuals with drug sensitive TB (T). (b) Individuals with MDR $(M)$. (c) Individuals with XDR $(X)$. Parameter values used are as given in Table 2.

(i) The model is locally asymptotically stable (LAS) $\mathscr{R}_{0}<$ 1 and unstable when $\mathscr{R}_{0}>1$.

(ii) The model exhibits in the presence of disease reinfection the phenomenon of backward bifurcation, where the stable disease-free coexists with a stable endemic equilibrium, when the associated reproduction number is less than unity.

(iii) As the isolation rate for each strain of TB increases, the total number of individuals infected with the particular strain of TB decreases.

(iv) Model (2) in the absence of disease reinfection is globally asymptotically stable (GAS) $\mathscr{R}_{0}<1$.

(v) The sensitivity analysis of the model shows that the dominant parameters for the drug sensitive $\mathrm{TB}$ are the disease progression rate $\left(\sigma_{T}\right)$, the recovery rate $\left(\gamma_{T}\right)$ from drug sensitive TB, the infectivity parameter $\left(\eta_{T}\right)$, the isolation rate $\left(\alpha_{T}\right)$ from drug sensitive TB class, fraction of fast progression rates and $\left(l_{T 1}\right.$ and $l_{T 2}$ ) into the drug sensitive TB class and lost to followup class, and the rate of lost to follow-up $\left(\phi_{T}\right)$. Similar parameters and return rates from lost to follow-up $\left(\psi_{M}\right.$ and $\left.\psi_{X}\right)$ are dominant for MDR- and XDRTB. The natural death rate $(\mu)$, although dominant in MDR- and XDR-TB, is however epidemiologically irrelevant.

(vi) Increase in isolation rate leads to increase in total number of individuals isolated with each TB strain resulting in decreases in the total number of individuals who are lost to follow-up. 


\section{Appendices}

\section{A. Proof of Theorem 6}

Proof. The proof is based on using the centre manifold theory [47], as described in [54]. It is convenient to make the following simplification and change of variables.
Let $S=x_{1}, E_{T}=x_{2}, T=x_{3}, E_{M}=x_{4}, M=x_{5}, E_{X}=$ $x_{6}, X=x_{7}, L_{T}=x_{8}, L_{M}=x_{9}, L_{X}=x_{10}, J=x_{11}$ and $R=x_{12}$, so that $N=x_{1}+x_{2}+x_{3}+x_{4}+x_{5}+$ $x_{6}+x_{7}+x_{8}+x_{9}+x_{10}+x_{11}+x_{12}$. Using the vector notation $\mathbf{x}=\left(x_{1}, x_{2}, x_{3}, x_{4}, x_{5}, x_{6}, x_{7}, x_{8}, x_{9}, x_{10}, x_{11}, x_{12}\right)^{T}$, model (2) can be written in the form $d \mathbf{x} / d t=\mathbf{m}(\mathbf{x})$, where $\mathbf{m}=\left(m_{1}, m_{2}, m_{3}, m_{4}, m_{5}, m_{6}, m_{7}, m_{8}, m_{9}, m_{10}, m_{11}, m_{12}\right)^{T}$, as follows:

$$
\begin{aligned}
& \frac{d x_{1}}{d t}=m_{1}=\pi-\frac{\left[\beta_{T}\left(x_{3}+\eta_{T} x_{8}\right)+\beta_{M}\left(x_{5}+\eta_{M} x_{9}\right)+\beta_{X} x_{7}\left(x_{7}+\eta_{X} x_{10}\right)\right] x_{1}}{\left(x_{1}+x_{2}+x_{3}+x_{4}+x_{5}+x_{6}+x_{7}+x_{8}+x_{9}+x_{10}+x_{11}+x_{12}\right)}-\mu x_{1}, \\
& \frac{d x_{2}}{d t}=m_{2}=\frac{\left(1-l_{T 1}-l_{T 2}\right) \beta_{T}\left(x_{3}+\eta_{T} x_{8}\right)\left(x_{1}+\varepsilon x_{12}\right)}{\left(x_{1}+x_{2}+x_{3}+x_{4}+x_{5}+x_{6}+x_{7}+x_{8}+x_{9}+x_{10}+x_{11}+x_{12}\right)}-g_{1} x_{2}, \\
& \frac{d x_{3}}{d t}=m_{3}=\frac{l_{T 1} \beta_{T}\left(x_{3}+\eta_{T} x_{8}\right)\left(x_{1}+\varepsilon x_{12}\right)}{\left(x_{1}+x_{2}+x_{3}+x_{4}+x_{5}+x_{6}+x_{7}+x_{8}+x_{9}+x_{10}+x_{11}+x_{12}\right)}+\sigma_{T} x_{2}+\psi_{T} x_{8}-g_{2} x_{3}, \\
& \frac{d x_{4}}{d t}=m_{4}=\frac{\left(1-l_{M 1}-l_{M 2}\right) \beta_{T}\left(x_{5}+\eta_{M} x_{9}\right)\left(x_{1}+\varepsilon x_{12}\right)}{\left(x_{1}+x_{2}+x_{3}+x_{4}+x_{5}+x_{6}+x_{7}+x_{8}+x_{9}+x_{10}+x_{11}+x_{12}\right)}-g_{3} x_{4}, \\
& \frac{d x_{5}}{d t}=m_{5}=\frac{l_{M 1} \beta_{T}\left(x_{5}+\eta_{M} x_{9}\right)\left(x_{1}+\varepsilon x_{12}\right)}{\left(x_{1}+x_{2}+x_{3}+x_{4}+x_{5}+x_{6}+x_{7}+x_{8}+x_{9}+x_{10}+x_{11}+x_{12}\right)}+\sigma_{m} x_{4}+\rho_{T} x_{3}+\psi_{m} x_{9}-g_{4} x_{5} \\
& \frac{d x_{6}}{d t}=m_{6}=\frac{\left(1-l_{X 1}-l_{X 2}\right) \beta_{X}\left(x_{7}+\eta_{X} x_{10}\right)\left(x_{1}+\varepsilon x_{12}\right)}{\left(x_{1}+x_{2}+x_{3}+x_{4}+x_{5}+x_{6}+x_{7}+x_{8}+x_{9}+x_{10}+x_{11}+x_{12}\right)}-g_{5} x_{6}, \\
& \frac{d x_{7}}{d t}=m_{7}=\frac{l_{X 1} \beta_{X}\left(x_{7}+\eta_{X} x_{10}\right)\left(x_{1}+\varepsilon x_{12}\right)}{\left(x_{1}+x_{2}+x_{3}+x_{4}+x_{5}+x_{6}+x_{7}+x_{8}+x_{9}+x_{10}+x_{11}+x_{12}\right)}+\sigma_{X} x_{6}+\rho_{M} x_{5}+\psi_{X} x_{10}-g_{6} x_{7}, \\
& \frac{d x_{8}}{d t}=m_{8}=\frac{l_{T 2} \beta_{T}\left(x_{3}+\eta_{T} x_{8}\right)\left(x_{1}+\varepsilon x_{12}\right)}{\left(x_{1}+x_{2}+x_{3}+x_{4}+x_{5}+x_{6}+x_{7}+x_{8}+x_{9}+x_{10}+x_{11}+x_{12}\right)}+\phi_{T} x_{3}-g_{7} x_{8}, \\
& \frac{d x_{9}}{d t}=m_{9}=\frac{l_{M 2} \beta_{M}\left(x_{5}+\eta_{M} x_{6}\right)\left(x_{1}+\varepsilon x_{12}\right)}{\left(x_{1}+x_{2}+x_{3}+x_{4}+x_{5}+x_{6}+x_{7}+x_{8}+x_{9}+x_{10}+x_{11}+x_{12}\right)}+\phi_{M} x_{5}-g_{8} x_{9}, \\
& \frac{d x_{10}}{d t}=m_{10}=\frac{l_{X 2} \beta_{X}\left(x_{7}+\eta_{T} x_{10}\right)\left(x_{1}+\varepsilon x_{12}\right)}{\left(x_{1}+x_{2}+x_{3}+x_{4}+x_{5}+x_{6}+x_{7}+x_{8}+x_{9}+x_{10}+x_{11}+x_{12}\right)}+\phi_{X} x_{7}-g_{9} x_{10}, \\
& \frac{d x_{11}}{d t}=m_{11}=\alpha_{T} x_{3}+\alpha_{M} x_{5}+\alpha_{M} x_{7}-g_{10} x_{11} \\
& \frac{d x_{12}}{d t}=m_{12}=\gamma_{T} x_{3}+\gamma_{M} x_{5}+\gamma_{X} x_{7}-g_{11} x_{12}-\frac{\varepsilon\left[\beta_{T}\left(x_{3}+\eta_{T} x_{8}\right)+\beta_{M}\left(x_{5}+\eta_{M} x_{9}\right)+\beta_{X}\left(x 7+\eta_{X} x_{10}\right)\right] x_{12}}{\left(x_{1}+x_{2}+x_{3}+x_{4}+x_{5}+x_{6}+x_{7}+x_{8}+x_{9}+x_{10}+x_{11}+x_{12}\right)} \text {. }
\end{aligned}
$$

The Jacobian of the transformed system (A.1), at the diseasefree equilibrium $\mathscr{E}_{1}$, is given by

$$
J\left(\mathscr{E}_{1}\right)=\left(J_{1} \mid J_{2}\right),
$$


where

$$
J_{1}=\left(\begin{array}{cccccc}
-\mu & 0 & -\beta_{T} & 0 & -\beta_{M} & 0 \\
0 & -g_{1} & l_{T 1} \beta_{T}+p_{T 1} \gamma_{T} & 0 & 0 & 0 \\
0 & \sigma_{T} & l_{T 2} \beta_{T}-g_{2} & 0 & 0 & 0 \\
0 & 0 & 0 & -g_{3} & l_{M 1} \beta_{M} & 0 \\
0 & 0 & p_{T 2} \gamma_{T} & \sigma_{M} & l_{M 2} \beta_{M}-g_{4} & 0 \\
0 & 0 & 0 & 0 & p_{M 1} \gamma_{M} & -g_{5} \\
0 & 0 & 0 & 0 & 0 & \sigma_{X} \\
0 & 0 & \left(1-l_{T 1}-l_{T 2}\right) \beta_{T}+\phi_{T} & 0 & 0 & 0 \\
0 & 0 & 0 & 0 & \left(1-l_{M 1}-l_{M 2}\right) \beta_{M}+\phi_{M} & 0 \\
0 & 0 & 0 & 0 & 0 & 0 \\
0 & 0 & \alpha_{T} & 0 & \alpha_{M} & 0 \\
0 & 0 & \left(1-p_{T 1}-p_{T 2}\right) \gamma_{T} & 0 & \left(1-p_{M 1}\right) \gamma_{M} & 0
\end{array}\right) \text {, }
$$

$J_{2}$

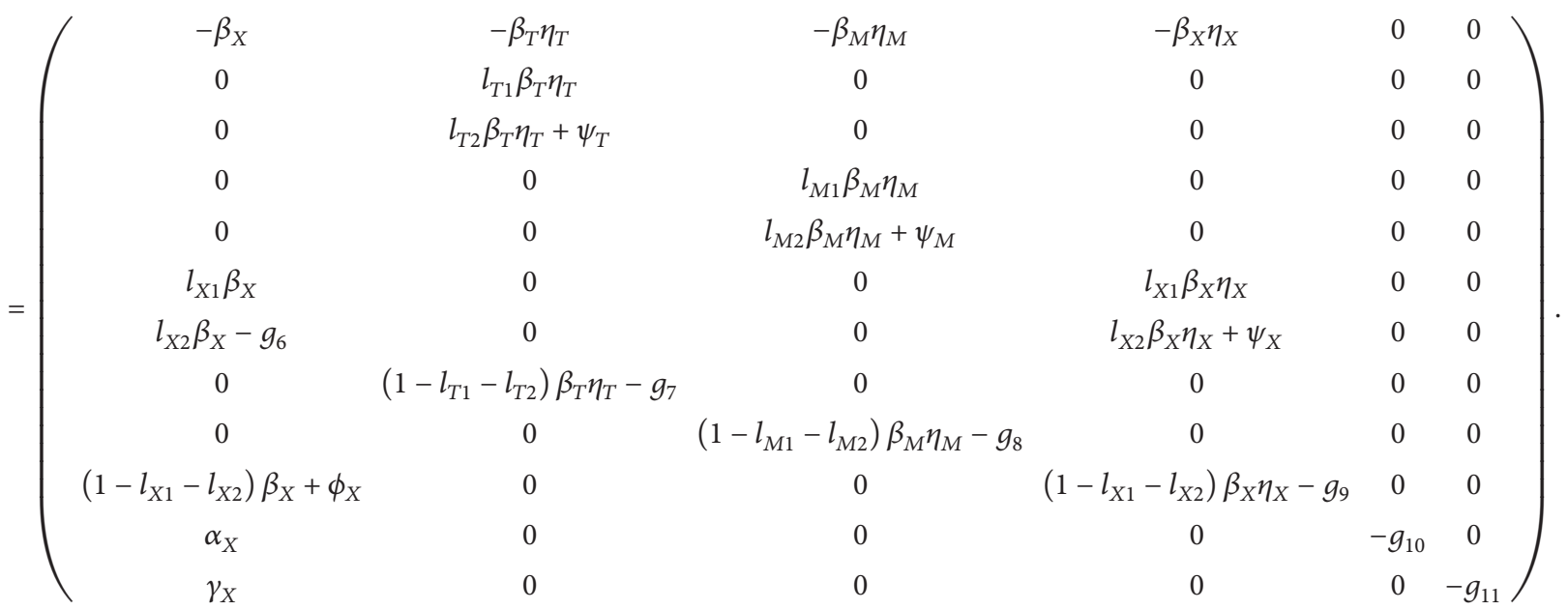

Consider the case when $\mathscr{R}_{0}=1$. Suppose, further, that $\beta_{T}$ is chosen as a bifurcation parameter. Solving (2) for $\beta_{T}$ from $\mathscr{R}_{0}=1$ gives $\beta_{T}=\beta_{T}^{*}$. The transformed system (A.1) at the DFE evaluated at $\beta_{T}=\beta_{T}^{*}$ has a simple zero eigenvalue (and all other eigenvalues having negative real parts). Hence, the centre manifold theory [47] can be used to analyze the dynamics of (A.1) near $\beta_{p}=\beta_{T}^{*}$. In particular, the theorem in [54] (see also $[36,47,48]$ ) is used (it is reproduced in the Appendix for convenience). To apply the theorem, the following computations are necessary (it should be noted that we are using $\beta_{T}$ instead of $\phi$ for the bifurcation parameter).

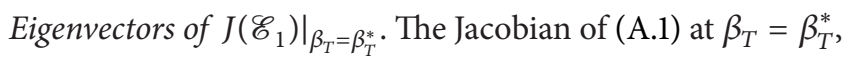
denoted by $\left.J\left(\mathscr{E}_{1}\right)\right|_{\beta_{T}=\beta_{T}^{*}}$, has a right eigenvector (associated with the zero eigenvalue) given by

$$
\begin{aligned}
& \mathbf{w}=\left(w_{1}, w_{2}, w_{3}, w_{4}, w_{5}, w_{6}, w_{7}, w_{8}, w_{9}, w_{10}, w_{11},\right. \\
& \left.w_{12}\right)^{T}
\end{aligned}
$$

where

$$
\begin{array}{rl}
w_{1} & =-\frac{1}{\mu}\left[\beta_{T} w_{3}+\beta_{M} w_{5}+\beta_{X} w_{7}+\beta_{T} \eta_{T} w_{8}\right. \\
& \left.+\beta_{M} \eta_{M} w_{9}+\beta_{X} \eta_{X} w_{10}\right] \\
w_{2} & =\frac{1}{g_{1}}\left\{\left[p_{T 1} \gamma_{T}+l_{T 1} \beta_{T}\right] w_{3}+l_{T 1} \beta_{T} \eta_{T} w_{8}\right\}, \\
w_{3} & >0 \\
w_{5}>0 & 0 \\
w_{4}= & \frac{1}{g_{3}}\left[p_{T 2} \gamma_{T} w_{3}+l_{M 1} \beta_{M} w_{5}+l_{M 1} \beta_{M} \eta_{M} w_{9}\right], \\
w_{6}= & \frac{1}{g_{5}}\left[p_{M 1} \gamma_{M} w_{5}+l_{X 1} \beta_{X} w_{7}+l_{X 1} \beta_{X} \eta_{X} w_{10}\right], \\
w_{7}>0 & 0 \\
w_{8}= & \frac{\left[\left(1-l_{T 1}-l_{T 2}\right) \beta_{T}+\phi_{T}\right] w_{3}}{\left[g_{7}-\left(1-l_{T 1}-l_{T 2}\right) \beta_{T} \eta_{T}\right]}
\end{array}
$$




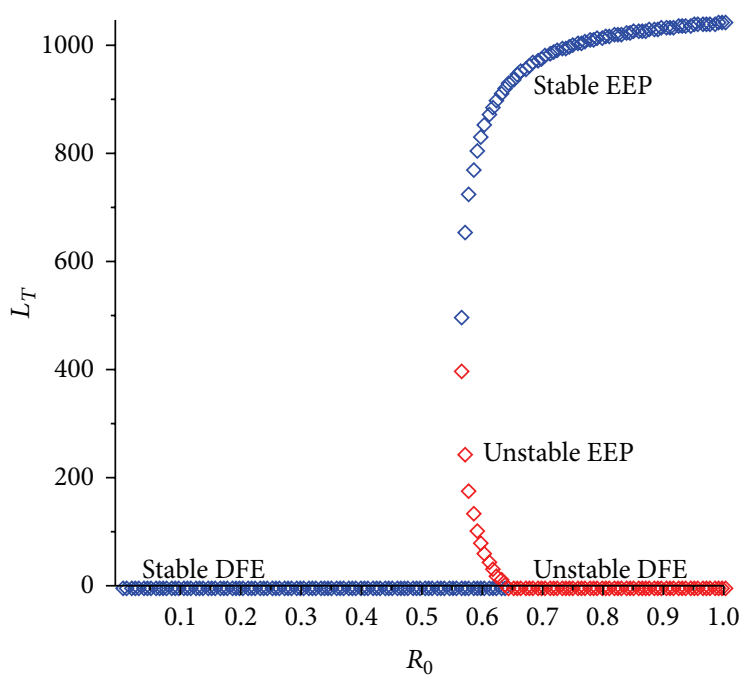

(a)

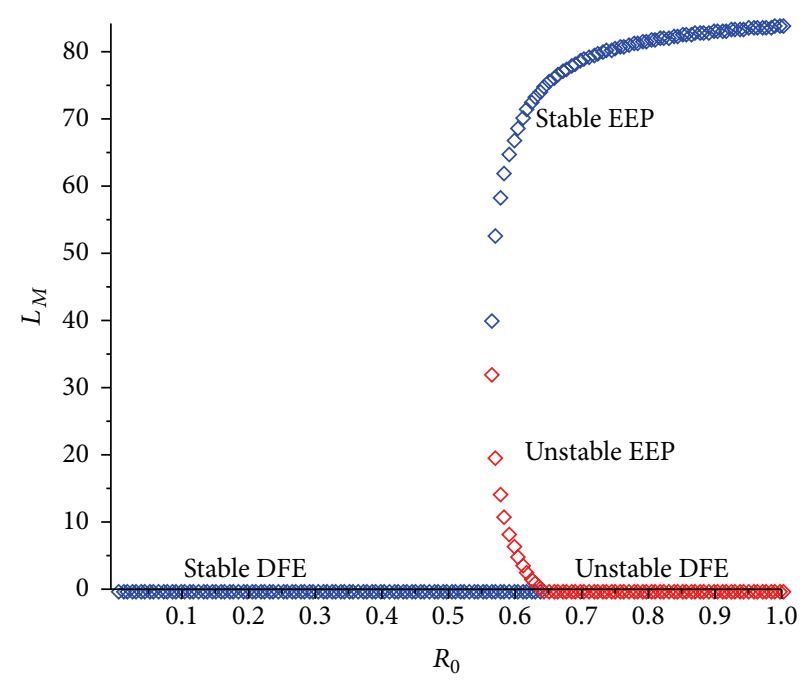

(b)

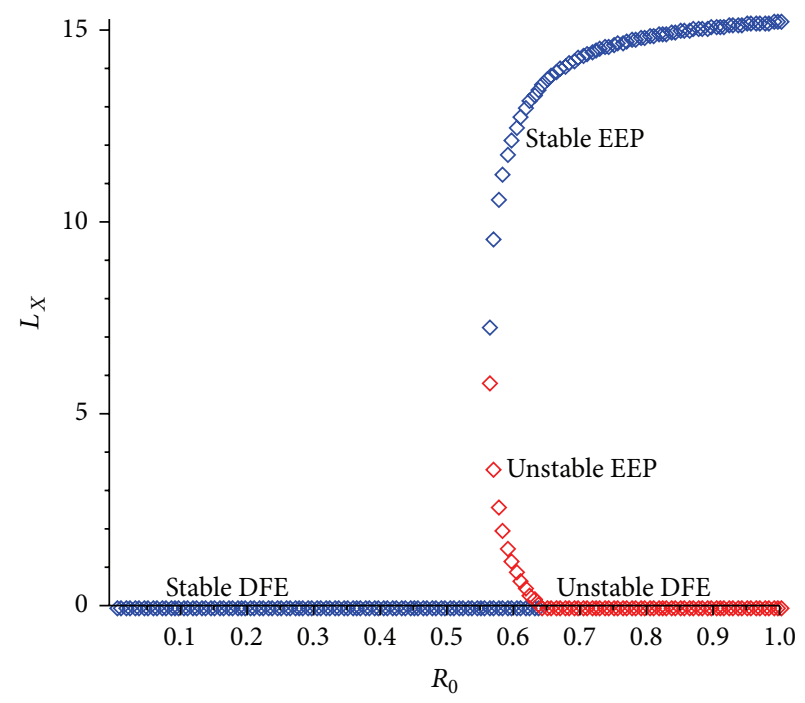

(c)

Figure 6: Backward bifurcation plot of model (2) as a function of time. (a) Individuals who are lost to follow-up with drug sensitive TB (T). (b) Individuals who are lost to follow-up with MDR $(M)$. (c) Individuals who are lost to follow-up with XDR $(X)$. Parameter values used are as given in Table 2.

$$
\begin{aligned}
& w_{9}=\frac{\left[\left(1-l_{M 1}-l_{M 2}\right) \beta_{M}+\phi_{M}\right] w_{5}}{\left[g_{8}-\left(1-l_{M 1}-l_{M 2}\right) \beta_{M} \eta_{M}\right]}, \\
& w_{10}=\frac{\left[\left(1-l_{X 1}-l_{X 2}\right) \beta_{X}+\phi_{X}\right] w_{7}}{\left(g_{9}-\left(1-l_{X 1}-l_{X 2}\right) \beta_{X} \eta_{X}\right)}, \\
& w_{11}=\frac{\left(\alpha_{M} w_{5}+\alpha_{X} w_{7}\right)}{g_{10}}, \\
& w_{12}=\frac{1}{g_{11}}\left[\left(1-p_{T 1}-p_{T 2}\right) \gamma_{T} w_{3}+\left(1-p_{M 1}\right) \gamma_{M} w_{5}\right. \\
& \left.+\gamma_{X} w_{7}\right] .
\end{aligned}
$$

Also, $\left.J\left(\mathscr{E}_{1}\right)\right|_{\beta_{T}=\beta_{T}^{*}}$ has a left eigenvector $\mathbf{v}=\left(v_{1}, v_{2}, v_{3}\right.$, $v_{4}, v_{5}, v_{6}, v_{7}, v_{8}, v_{9}, v_{10}, v_{11}, v_{12}$ ) (associated with the zero eigenvalue), where

$$
\begin{aligned}
& v_{1}=0, \\
& v_{2}=\frac{\sigma_{T} v_{3}}{g_{1}}, \\
& v_{4}=\frac{\sigma_{M} v_{4}}{g_{3}}, \\
& v_{6}=\frac{\sigma_{X} v_{7}}{g_{5}},
\end{aligned}
$$

$$
v_{3}>0 \text {, }
$$




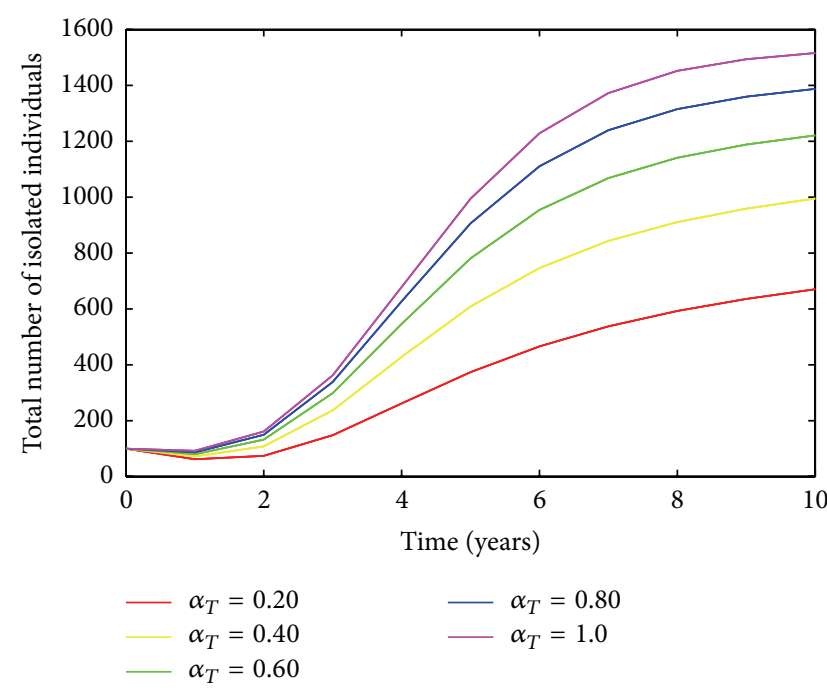

(a)

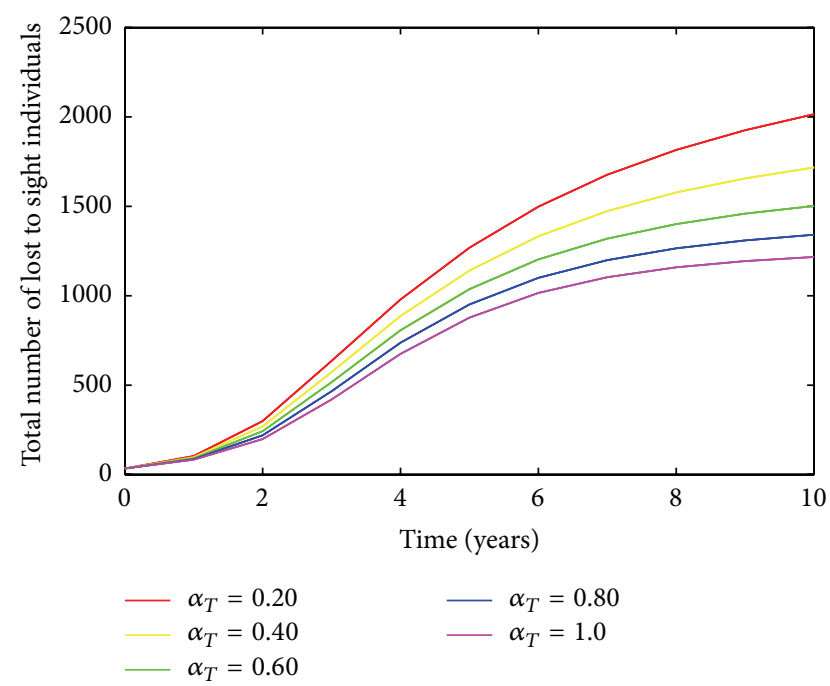

(b)

Figure 7: Simulation of model (2) as a function of time varying $\alpha_{T}$ for (a) total number of isolated individuals and (b) total number of individuals who are lost to follow-up. Parameter values used are as given in Table 2.

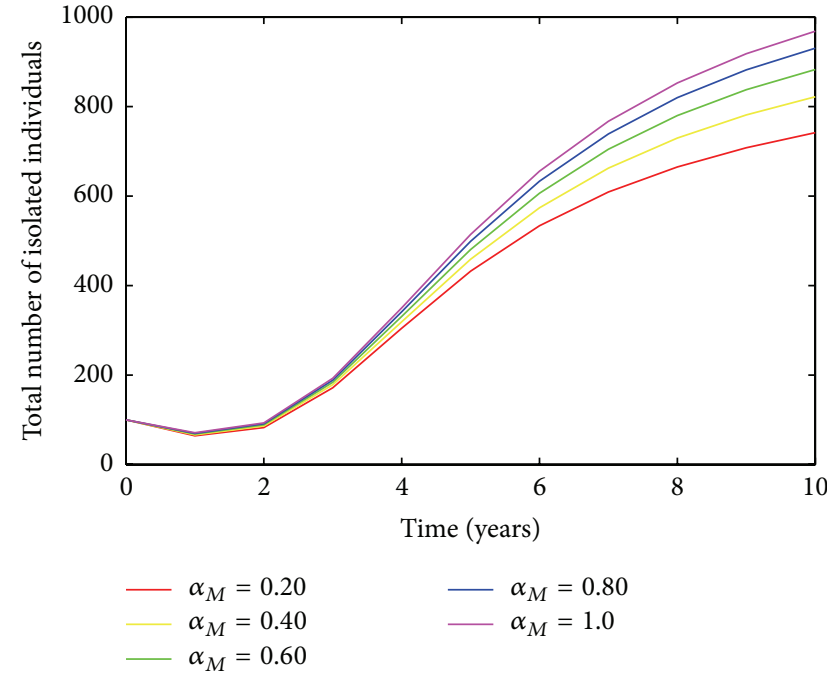

(a)

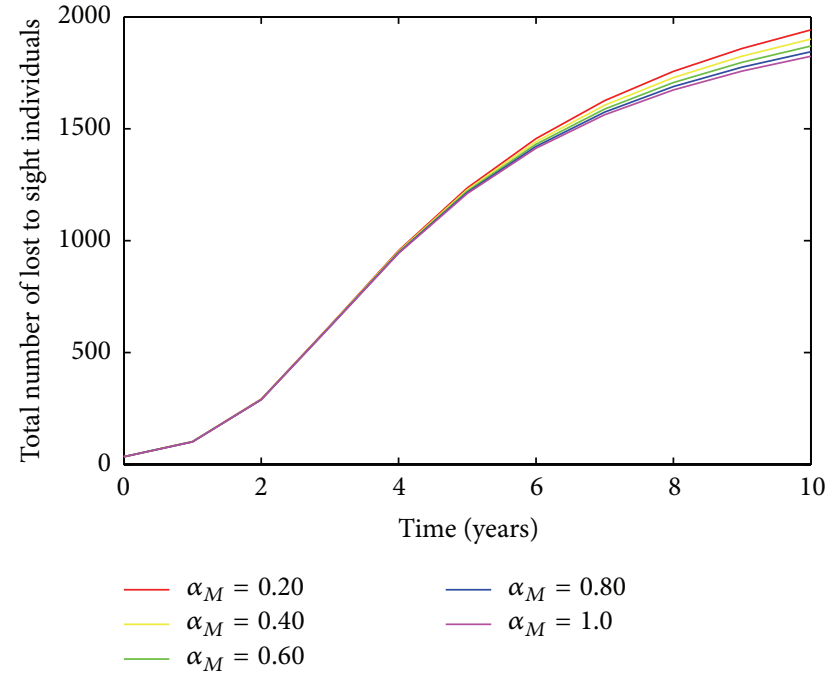

(b)

Figure 8: Simulation of model (2) as a function of time varying $\alpha_{M}$ for (a) total number of isolated individuals and (b) total number of individuals who are lost to follow-up. Parameter values used are as given in Table 2.

$$
\begin{aligned}
v_{7} & >0 \\
v_{5} & =\frac{1}{\left(g_{4}-l_{M 2} \beta_{M}\right)}\left[l_{M 1} \beta_{M} v_{4}+p_{M 1} \gamma_{M} v_{6}\right. \\
& \left.+\left[\left(1-l_{M 1}-l_{M 2}\right) \beta_{M}+\phi_{M}\right] v_{9}\right], \\
v_{8} & =\frac{1}{\left[g_{7}-\left(1-l_{T 1}-l_{T 2}\right) \beta_{T} \eta_{T}\right]}\left[l_{T 1} \beta_{T} \eta_{T} v_{2}\right. \\
& \left.+\left(l_{T 2} \beta_{T} \eta_{T}+\psi_{T}\right) v_{3}\right], \\
v_{9} & =\frac{1}{\left(g_{8}-\left(1-l_{M 1}-l_{M 2}\right) \beta_{M} \eta_{M}\right)}\left[l_{M 1} \beta_{M} \eta_{M} v_{4}\right.
\end{aligned}
$$

$$
\begin{aligned}
& \left.+\left(l_{M 2} \beta_{M} \eta_{M}+\psi_{M}\right) v_{5}\right], \\
v_{10} & =\frac{1}{\left(g_{9}-\left(1-l_{X 1}-l_{X 2}\right) \beta_{X} \eta_{X}\right)}\left[l_{X 1} \beta_{X} \eta_{X} v_{6}\right. \\
& \left.+\left(l_{X 2} \beta_{X} \eta_{X}+\psi_{X}\right) v_{7}\right] \\
v_{11} & =0 \\
v_{12} & =0 .
\end{aligned}
$$

Computations of Bifurcation Coefficients $a$ and $b$. The application of the theorem (given in the Appendix) entails the 
computation of two bifurcation coefficients $a$ and $b$. It can be shown, after some algebraic manipulations, that

$$
\begin{aligned}
a & =v_{2} \sum_{i, j=1}^{12} w_{i} w_{j} \frac{\partial^{2} f_{2}}{\partial x_{i} \partial x_{j}}+v_{3} \sum_{i, j=1}^{12} w_{i} w_{j} \frac{\partial^{2} f_{3}}{\partial x_{i} \partial x_{j}} \\
& +v_{4} \sum_{i, j=1}^{12} w_{i} w_{j} \frac{\partial^{2} f_{4}}{\partial x_{i} \partial x_{j}}+v_{5} \sum_{i, j=1}^{12} w_{i} w_{j} \frac{\partial^{2} f_{5}}{\partial x_{i} \partial x_{j}} \\
& +v_{6} \sum_{i, j=1}^{12} w_{i} w_{j} \frac{\partial^{2} f_{6}}{\partial x_{i} \partial x_{j}}+v_{7} \sum_{i, j=1}^{12} w_{i} w_{j} \frac{\partial^{2} f_{7}}{\partial x_{i} \partial x_{j}} \\
& =\frac{2}{x_{1}}\left(-w_{2}-w_{3}-w_{4}-w_{5}-w_{6}-w_{7}-w_{8}-w_{9}\right. \\
& \left.-w_{10}-w_{11}-w_{12}+w_{12} \varepsilon\right)\left\{\beta_{T}\left(w_{3}+w_{8} \eta_{T}\right)\right. \\
& \cdot\left[l_{T 1} v_{2}+l_{T 2} v_{3}+\left(1-l_{T 1}-l_{T 2}\right) v_{8}\right] \\
& +\beta_{M}\left(w_{5}+w_{9} \eta_{M}\right) \\
& \cdot\left[l_{M 1} v_{4}+l_{M 2} v_{5}+\left(1-l_{M 1}-l_{M 2}\right) v_{9}\right] \\
& +\beta_{X}\left(w_{7}+w_{10} \eta_{X}\right) \\
& \left.\cdot\left[l_{X 1} v_{6}+l_{X 2} v_{7}+\left(1-l_{X 1}-l_{X 2}\right) v_{10}\right]\right\} .
\end{aligned}
$$

Furthermore,

$$
\begin{aligned}
b & =v_{2} \sum_{i=1}^{12} w_{i} \frac{\partial^{2} f_{2}}{\partial x_{i} \partial \beta_{p}^{*}}+v_{3} \sum_{i=1}^{12} w_{i} \frac{\partial^{2} f_{3}}{\partial x_{i} \partial \beta_{p}^{*}} \\
& =\left(w_{3}+w_{8} \eta_{T}\right)\left[l_{T 1} v_{2}+l_{T 2} v_{3}+\left(1-l_{T 1}-l_{T 2}\right) v_{8}\right] \\
& >0 .
\end{aligned}
$$

Hence, it follows from Theorem 4.1 of [54] that the transformed model (A.1) (or, equivalently, (2)) undergoes backward bifurcation at $\mathscr{R}_{0}=1$ whenever the following inequality holds:

$$
a>0 \text {. }
$$

It is worth noting that if $\varepsilon=0$ (i.e., reinfection of recovered individuals does not occur), the bifurcation coefficient, $a$, given in (A.7), reduces to

$$
\begin{aligned}
a= & -\frac{2}{x_{1}}\left(w_{2}+w_{3}+w_{4}+w_{5}+w_{6}+w_{7}+w_{8}+w_{9}\right. \\
& \left.+w_{10}+w_{11}\right)\left\{\beta_{T}\left(w_{3}+w_{8} \eta_{T}\right)\right. \\
& \cdot\left[l_{T 1} v_{2}+l_{T 2} v_{3}+\left(1-l_{T 1}-l_{T 2}\right) v_{8}\right] \\
& +\beta_{M}\left(w_{5}+w_{9} \eta_{M}\right) \\
& \cdot\left[l_{M 1} v_{4}+l_{M 2} v_{5}+\left(1-l_{M 1}-l_{M 2}\right) v_{9}\right]
\end{aligned}
$$

$$
\begin{aligned}
& +\beta_{X}\left(w_{7}+w_{10} \eta_{X}\right) \\
& \left.\cdot\left[l_{X 1} v_{6}+l_{X 2} v_{7}+\left(1-l_{X 1}-l_{X 2}\right) v_{10}\right]\right\} .
\end{aligned}
$$

It follows from (A.10) that the bifurcation coefficient $a<0$ (ruling out backward bifurcation in this case, in line with Theorem 4.1 in [54]). Thus, this study shows that the backward bifurcation phenomenon of model (A.1) is caused by the reinfection of the recovered individuals in the population.

\section{B. Proof of Theorem 7}

Proof. The proof is based on using a comparison theorem. The equations for the infected components of model (2), with $\varepsilon=0$, can be rewritten as

$$
\left.\begin{array}{c}
\frac{d E_{T}(t)}{d t} \\
\frac{d T(t)}{d t} \\
\frac{d E_{M}(t)}{d t} \\
\frac{d M(t)}{d t} \\
\frac{d E_{X}(t)}{d t} \\
\frac{d X(t)}{d t} \\
\frac{d L_{T}(t)}{d t} \\
\frac{d L_{M}(t)}{d t} \\
\frac{d L_{X}(t)}{d t} \\
\frac{d J(t)}{d t}
\end{array}\right)=(F-V)\left(\begin{array}{c}
E_{T}(t) \\
T(t) \\
E_{M}(t) \\
M(t) \\
E_{X}(t) \\
X(t) \\
L_{T}(t) \\
L_{M}(t) \\
L_{X}(t) \\
J(t)
\end{array}\right)
$$


where $P=1-S$, the matrices $F$ and $V$ are as given in where Section 2.2, and $Q$ is nonnegative matrices given, respectively, by

$$
Q=\left[Q_{1} \mid Q_{2}\right]
$$

$$
\begin{aligned}
& Q_{1}=\left(\begin{array}{ccccc}
0 & l_{T 1} \beta_{T} & 0 & 0 & 0 \\
0 & l_{T 2} \beta_{T} & 0 & 0 & 0 \\
0 & 0 & 0 & l_{M 1} \beta_{M} & 0 \\
0 & 0 & 0 & l_{M 2} \beta_{M} & 0 \\
0 & 0 & 0 & 0 & 0 \\
0 & 0 & 0 & 0 & 0 \\
0 & \left(1-l_{T 1}-l_{T 2}\right) \beta_{T} & 0 & 0 & 0 \\
0 & 0 & 0 & \left(1-l_{M 1}-l_{M 2}\right) \beta_{M} & 0 \\
0 & 0 & 0 & 0 & 0 \\
0 & 0 & 0 & 0 & 0
\end{array}\right),
\end{aligned}
$$

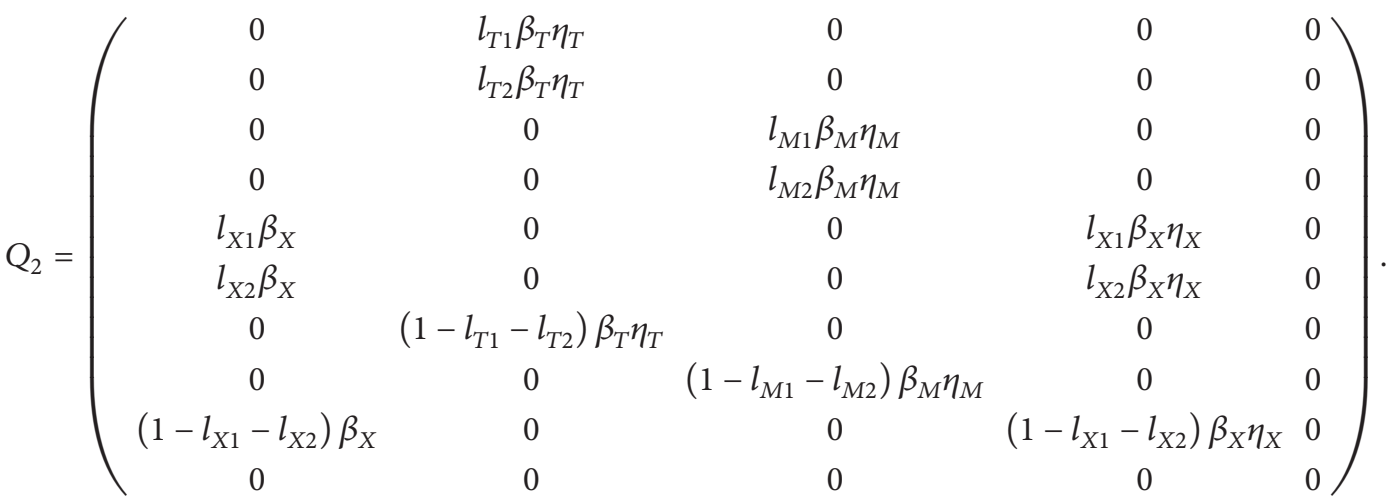

Thus, since $S(t) \leq N(t)$ in $\Phi$ for all $t \geq 0$, it follows from (B.1) that

$$
\left(\begin{array}{c}
\frac{d E_{T}(t)}{d t} \\
\frac{d T(t)}{d t} \\
\frac{d E_{M}(t)}{d t} \\
\frac{d M(t)}{d t} \\
\frac{d E_{X}(t)}{d t} \\
\frac{d X(t)}{d t} \\
\frac{d L_{T}(t)}{d t} \\
\frac{d L_{M}(t)}{d t} \\
\frac{d L_{X}(t)}{d t} \\
\frac{d J(t)}{d t}
\end{array}\right) \leq(F-V)\left(\begin{array}{c}
E_{T}(t) \\
T(t) \\
E_{M}(t) \\
M(t) \\
E_{X}(t) \\
X(t) \\
L_{T}(t) \\
L_{M}(t) \\
L_{X}(t) \\
J(t)
\end{array}\right)
$$

Using the fact that the eigenvalues of the matrix $F-V$ all have negative real parts (see the local stability result given in Lemma 3, where $\rho\left(F V^{-1}\right)<1$ if $\mathscr{R}_{0}<1$, which is equivalent to $F-V$ having eigenvalues with negative real parts when $\mathscr{R}_{0}<1$ [36]), it follows that the linearized differential inequality system (B.4) is stable whenever $\mathscr{R}_{0}<$ 1. Consequently, by comparison of theorem [34] (Theorem 1.5.2, p. 31),

$$
\begin{gathered}
\left(E_{T}(t), T(t), L_{T}(t), E_{M}(t), M(t), L_{M}(t), E_{X}(t),\right. \\
\left.X(t), L_{X}(t), J(t)\right) \longrightarrow(0,0,0,0,0,0,0,0,0,0),
\end{gathered}
$$

$$
\text { as } t \longrightarrow \infty \text {. }
$$

Substituting $E_{T}=T=L_{T}=E_{M}=M=L_{M}=E_{X}=X=$ $L_{X}=J=0$ into the equations of $S$ and $R$ in model (2), and noting that $\varepsilon=0$, gives $S(t) \rightarrow S^{*}, R(t) \rightarrow 0$ as $t \rightarrow \infty$. Thus, in summary,

$$
\begin{aligned}
& \left(S(t), E_{T}(t), T(t), L_{T}(t), E_{M}(t), M(t), L_{M}(t),\right. \\
& \left.\quad E_{X}(t), X(t), L_{X}(t), J(t), R(t)\right) \longrightarrow\left(S^{*}, 0,0,0,0,\right. \\
& \quad 0,0,0,0,0,0,0)
\end{aligned}
$$


as $t \rightarrow \infty$. Hence, the DFE $\left(\mathscr{E}_{0}\right)$ of model (2), with $\varepsilon=0$, is GAS in $\Phi$ if $\widetilde{R}_{0}<1$.

\section{Conflict of Interests}

The authors declare that there is no conflict of interests regarding the publication of this paper.

\section{References}

[1] Centers for Disease Control, Multidrug-Resistant TB Fact Sheet, 2013, http://www.cdc.gov/tb/publications/factsheets/drtb/mdrtb .htm.

[2] World Health Organization, Tuberculosis Fact Sheet, World Health Organization, Geneva, Switzerland, 2015, http://www .who.int/mediacentre/factsheets/fs104/en/.

[3] J. R. Andrews, N. S. Shah, N. Gandhi, T. Moll, and G. Friedland, "Multidrug-resistant and extensively drug-resistant tuberculosis: implications for the HIV epidemic and antiretroviral therapy rollout in South Africa," Journal of Infectious Diseases, vol. 196, no. 3, pp. S482-S490, 2007.

[4] World Health Organization, "Global tuberculosis report 2014," http://www.who.int/tb/publications/global_report/gtbr14_executive_summary.pdf.

[5] S. Basu and A. P. Galvani, "The transmission and control of XDR TB in South Africa: an operations research and mathematical modelling approach," Epidemiology \& Infection, vol. 136, no. 12, pp. 1585-1598, 2008.

[6] J. Hughes, H. Cox, and N. Ford, "Sanatoria for drug-resistant tuberculosis: an outdated response," The Lancet, vol. 379, no. 9832, pp. 2148-2149, 2012.

[7] K. Dheda and G. B. Migliori, "The global rise of extensively drug-resistant tuberculosis: is the time to bring back sanatoria now overdue?" The Lancet, vol. 379, no. 9817, pp. 773-775, 2012.

[8] K. R. Jacobson, D. B. Tierney, C. Y. Jeon, C. D. Mitnick, and M. B. Murray, "Treatment outcomes among patients with extensively drug-resistant tuberculosis: systematic review and meta-analysis," Clinical Infectious Diseases, vol. 51, no. 1, pp. 614, 2010.

[9] P. Sampathkumar, "Dealing with threat of drug-resistant tuberculosis: background information for interpreting the Andrew Speaker and related cases," Mayo Clinic Proceedings, vol. 82, no. 7, pp. 799-802, 2007.

[10] American Lung Association, Multidrug-Resistant Tuberculosis (MDR-TB) Fact Sheet, 2013, http://www.lung.org/lung-disease/ tuberculosis/factsheets/multidrug-resistant.html.

[11] B. Song, C. Castillo-Chavez, and J. P. Aparicio, “Tuberculosis models with fast and slow dynamics: the role of close and casual contacts," Mathematical Biosciences, vol. 180, pp. 187-205, 2002.

[12] R. I. Hickson, G. N. Mercer, and K. M. Lokuge, "A metapopulation model of tuberculosis transmission with a case study from high to low burden areas," PLoS ONE, vol. 7, no. 4, Article ID e34411, 2012.

[13] Y. Liu, Z. Sun, G. Sun et al., "Modeling transmission of tuberculosis with MDR and undetected cases," Discrete Dynamics in Nature and Society, vol. 2011, Article ID 296905, 12 pages, 2011.

[14] C. Castillo-Chavez and Z. Feng, "To treat or not to treat: the case of tuberculosis," Journal of Mathematical Biology, vol. 35, no. 6, pp. 629-656, 1997.
[15] T. Cohen and M. Murray, "Modeling epidemics of multidrugresistant $M$. tuberculosis of heterogeneous fitness," Nature Medicine, vol. 10, no. 10, pp. 1117-1121, 2004.

[16] M. G. M. Gomes, A. O. Franco, M. C. Gomes, and G. F. Medley, "The reinfection threshold promotes variability in tuberculosis epidemiology and vaccine efficacy," Proceedings of the Royal Society B: Biological Sciences, vol. 271, no. 1539, pp. 617-623, 2004.

[17] T. C. Porco and S. M. Blower, "Quantifying the intrinsic transmission dynamics of tuberculosis," Theoretical Population Biology, vol. 54, no. 2, pp. 117-132, 1998.

[18] E. Jung, S. Lenhart, and Z. Feng, "Optimal control of treatments in a two-strain tuberculosis model," Discrete and Continuous Dynamical Systems B, vol. 2, no. 4, pp. 473-482, 2002.

[19] S. M. Blower, A. R. McLean, T. C. Porco et al., "The intrinsic transmission dynamics of tuberculosis epidemics," Nature Medicine, vol. 1, no. 8, pp. 815-821, 1995.

[20] T. Cohen, C. Colijn, B. Finklea, and M. Murray, "Exogenous re-infection and the dynamics of tuberculosis epidemics: local effects in a network model of transmission," Journal of the Royal Society Interface, vol. 4, no. 14, pp. 523-531, 2007.

[21] Z. Feng, W. Huang, and C. Castillo-Chavez, "On the role of variable latent periods in mathematical models for tuberculosis," Journal of Dynamics and Differential Equations, vol. 13, no. 2, pp. 425-452, 2001.

[22] Y. Emvudu, R. Demasse, and D. Djeudeu, "Optimal control of the lost to follow up in a tuberculosis model," Computational and Mathematical Methods in Medicine, vol. 2011, Article ID 398476, 12 pages, 2011.

[23] S. Bowong, Y. Emvudu, D. P. Moualeu, and J. J. Tewa, "Mathematical properties of a tuberculosis model with two differential infectivity and N latent classes," Journal of Nonlinear Systems and Applications, vol. 1, pp. 13-26, 2010.

[24] M. W. Borgdorff, "New measurable indicator for tuberculosis case detection," Emerging Infectious Diseases, vol. 10, no. 9, 2004.

[25] C. Dye, G. P. Garnett, K. Sleeman, and B. G. Williams, "Prospects for worldwide tuberculosis control under the WHO DOTS strategy. Directly observed short-course therapy," The Lancet, vol. 352, no. 9144, pp. 1886-1891, 1998.

[26] P. M. Sutton, M. Nicas, and R. J. Harrison, "Tuberculosis isolation: comparison of written procedures and actual practices in three California hospitals," Infection Control and Hospital Epidemiology, vol. 21, no. 1, pp. 28-32, 2000.

[27] S. E. Weis, P. C. Slocum, F. X. Blais et al., "The effect of directly observed therapy on the rates of drug resistance and relapse in tuberculosis," The New England Journal of Medicine, vol. 330, no. 17, pp. 1179-1184, 1994.

[28] J. Frith, "History of tuberculosis. Part 2-the sanatoria and the discoveries of the Tubercle bacillus," Journal of Military and Veterans' Health, vol. 22, no. 2, pp. 36-41, 2014.

[29] M. R. O’Donnell, N. Padayatchi, C. Kvasnovsky, L. Werner, I. Master, and C. R. Horsburgh Jr., "Treatment outcomes for extensively drug-resistant tuberculosis and HIV co-infection," Emerging Infectious Diseases, vol. 19, no. 3, pp. 416-424, 2013.

[30] D. P. Moualeu, S. Bowong, and Y. Emvudu, "Global stability of a tuberculosis model with $\mathrm{N}$ latent classes," Journal of Applied Mathematics \& Informatics, vol. 29, no. 5-6, pp. 1097-1115, 2011.

[31] S. Bowong and J. J. Tewa, "Mathematical analysis of a tuberculosis model with differential infectivity," Communications in Nonlinear Science and Numerical Simulation, vol. 14, no. 11, pp. 4010-4021, 2009. 
[32] D. P. Moualeu, M. Weiser, R. Ehrig, and P. Deuflhard, "Optimal control for a tuberculosis model with undetected cases in Cameroon," Communications in Nonlinear Science and Numerical Simulation, vol. 20, no. 3, pp. 986-1003, 2015.

[33] H. R. Thieme, Mathematics in Population Biology, Princeton University Press, 2003.

[34] V. Lakshmikantham, S. Leela, and A. A. Martynyuk, Stability Analysis of Nonlinear Systems, Marcel Dekker, New York, NY, USA, 1989.

[35] H. W. Hethcote, "The mathematics of infectious diseases," SIAM Review, vol. 42, no. 4, pp. 599-653, 2000.

[36] P. van den Driessche and J. Watmough, "Reproduction numbers and sub-threshold endemic equilibria for compartmental models of disease transmission," Mathematical Biosciences, vol. 180, pp. 29-48, 2002.

[37] R. M. Anderson and R. M. May, Infectious Diseases of Humans, Oxford University Press, Oxford, UK, 1991.

[38] O. Diekmann, J. A. Heesterbeek, and J. A. Metz, "On the definition and computation of the basic reproduction ratio $R_{0}$ in models for infectious diseases in heterogeneous populations," Journal of Mathematical Biology, vol. 28, no. 4, pp. 365-382, 1990.

[39] S. M. Blower and H. Dowlatabadi, "Sensitivity and uncertainty analysis of complex models of disease transmission: an HIV model, as an example," International Statistical Review, vol. 62, no. 2, pp. 229-243, 1994.

[40] S. Marino, I. B. Hogue, C. J. Ray, and D. E. Kirschner, "A methodology for performing global uncertainty and sensitivity analysis in systems biology," Journal of Theoretical Biology, vol. 254, no. 1, pp. 178-196, 2008.

[41] M. D. McKay, R. J. Beckman, and W. J. Conover, "A comparison of three methods for selecting values of input variables in the analysis of output from a computer code," Technometrics, vol. 21, no. 2, pp. 239-245, 1979.

[42] R. G. McLeod, J. F. Brewster, A. B. Gumel, and D. A. Slonowsky, "Sensitivity and uncertainty analyses for a SARS model with time-varying inputs and outputs," Mathematical Biosciences and Engineering, vol. 3, no. 3, pp. 527-544, 2006.

[43] S.-F. H. Schmitz, "Effects of treatment or/and vaccination on HIV transmission in homosexuals with genetic heterogeneity," Mathematical Biosciences, vol. 167, no. 1, pp. 1-18, 2000.

[44] F. B. Agusto and A. B. Gumel, "Qualitative dynamics of lowlyand highly-pathogenic avian influenza strains," Mathematical Biosciences, vol. 243, no. 2, pp. 147-162, 2013.

[45] F. B. Agusto, A. B. Gumel, S. Lenhart, and A. Odoi, "Mathematical analysis of a model for the transmission dynamics of bovine tuberculosis," Mathematical Methods in the Applied Sciences, vol. 34, no. 15, pp. 1873-1887, 2011.

[46] F. Brauer, "Backward bifurcations in simple vaccination models," Journal of Mathematical Analysis and Applications, vol. 298, no. 2, pp. 418-431, 2004.

[47] J. Carr, Applications of Centre Manifold Theory, vol. 35, Springer, New York, NY, USA, 1981.

[48] J. Dushoff, W. Huang, and C. Castillo-Chavez, "Backwards bifurcations and catastrophe in simple models of fatal diseases," Journal of Mathematical Biology, vol. 36, no. 3, pp. 227-248, 1998.

[49] E. H. Elbasha and A. B. Gumel, "Theoretical assessment of public health impact of imperfect prophylactic HIV-1 vaccines with therapeutic benefits," Bulletin of Mathematical Biology, vol. 68 , no. 3, pp. 577-614, 2006.
[50] S. M. Garba and A. B. Gumel, "Effect of cross-immunity on the transmission dynamics of two strains of dengue," International Journal of Computer Mathematics, vol. 87, no. 10, pp. 2361-2384, 2010.

[51] S. M. Garba, A. B. Gumel, and M. R. Abu Bakar, "Backward bifurcations in dengue transmission dynamics," Mathematical Biosciences, vol. 215, no. 1, pp. 11-25, 2008.

[52] O. Sharomi, C. N. Podder, A. B. Gumel, E. H. Elbasha, and J. Watmough, "Role of incidence function in vaccine-induced backward bifurcation in some HIV models," Mathematical Biosciences, vol. 210, no. 2, pp. 436-463, 2007.

[53] O. Sharomi, C. N. Podder, A. B. Gumel, and B. Song, "Mathematical analysis of the transmission dynamics of HIV/TB co-infection in the presence of treatment," Mathematical Biosciences and Engineering, vol. 5, no. 1, pp. 145-174, 2008.

[54] C. Castillo-Chavez and B. Song, "Dynamical models of tuberculosis and their applications," Mathematical Biosciences and Engineering, vol. 1, no. 2, pp. 361-404, 2004. 


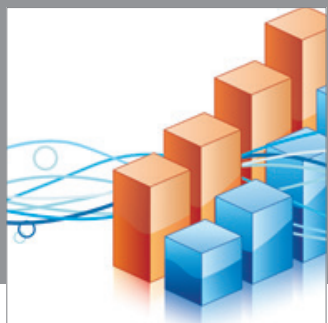

Advances in

Operations Research

mansans

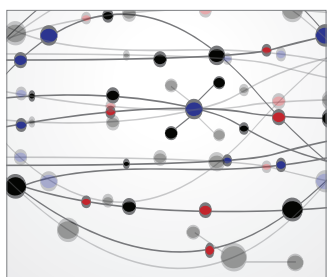

The Scientific World Journal
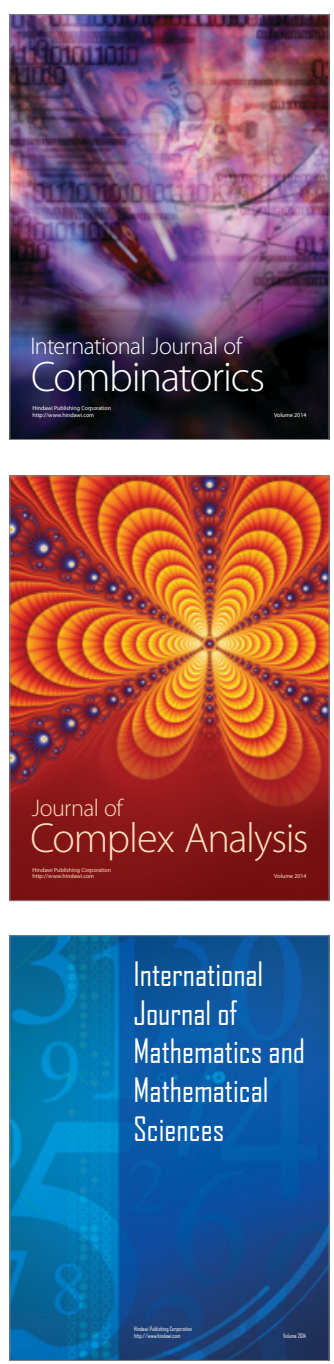
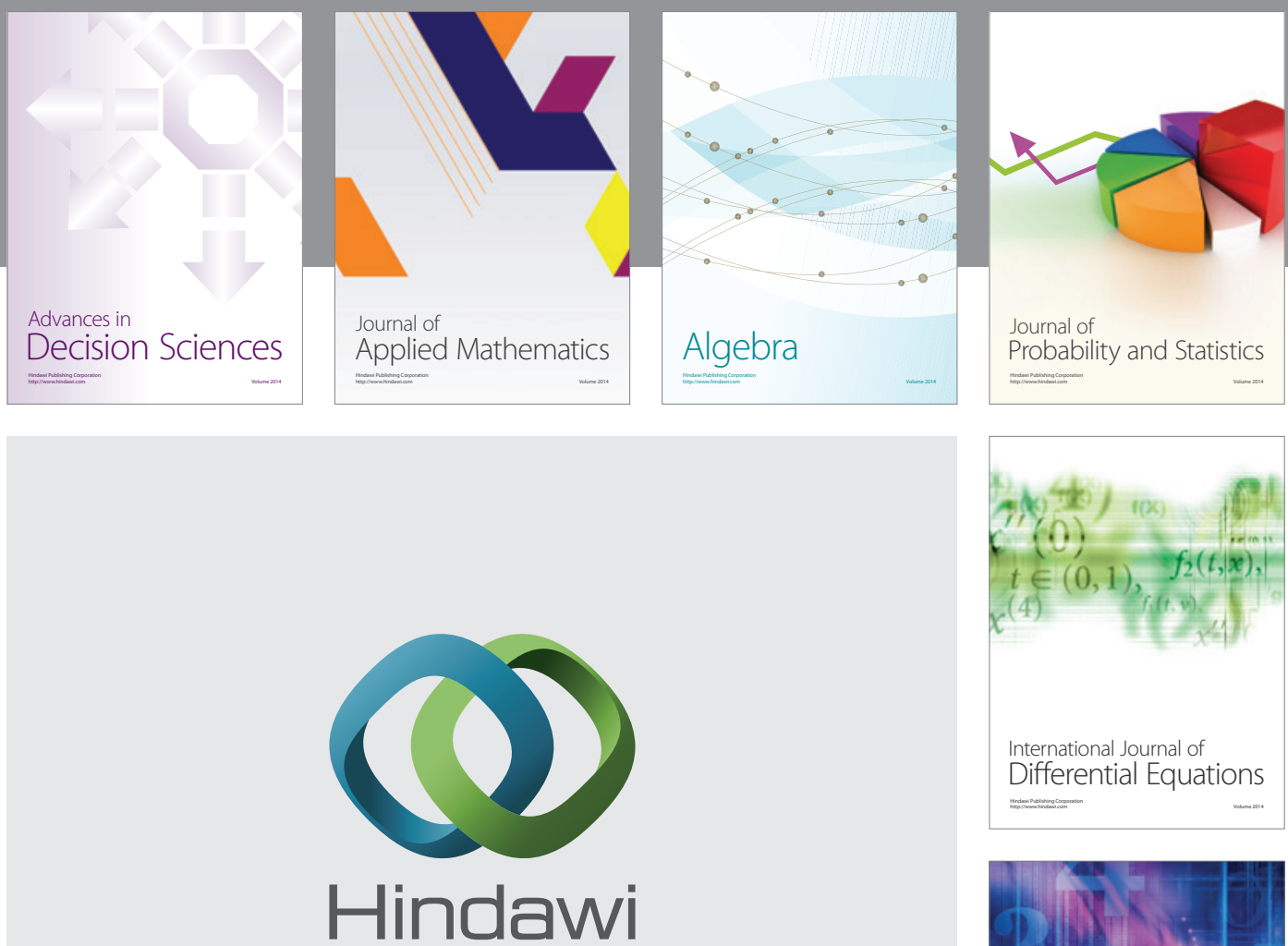

Submit your manuscripts at http://www.hindawi.com
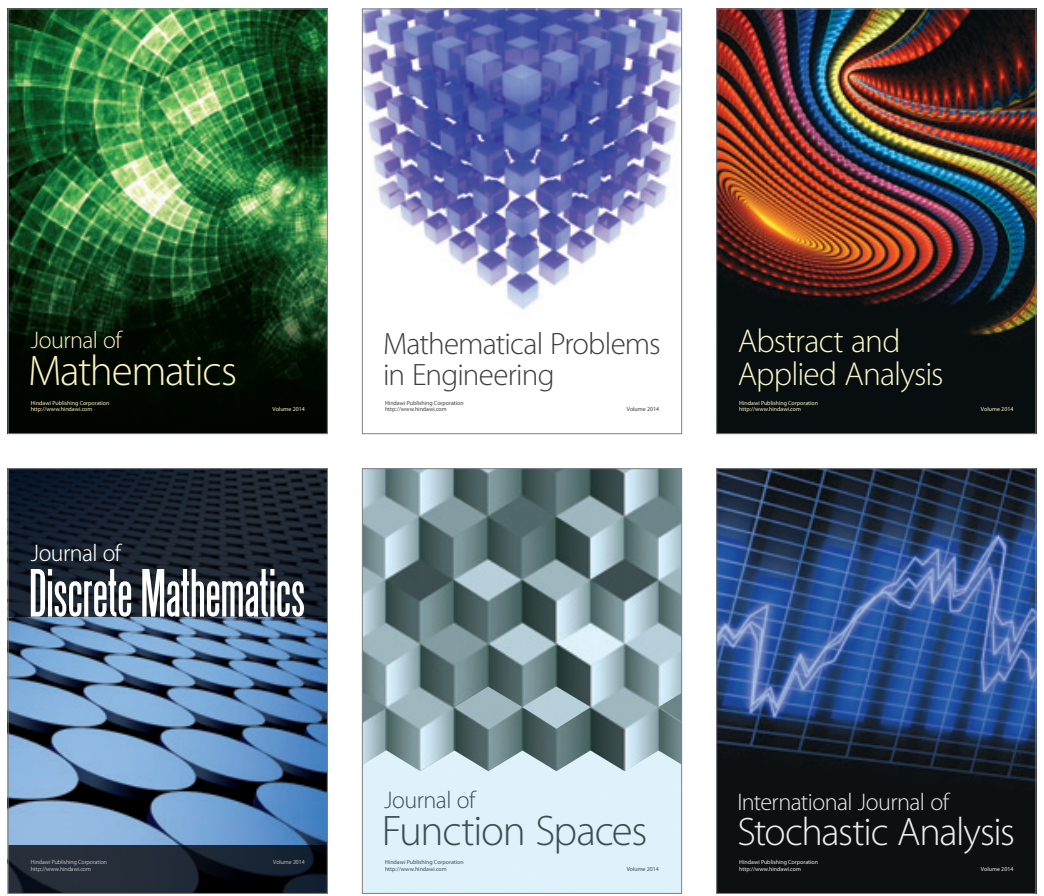

Journal of

Function Spaces

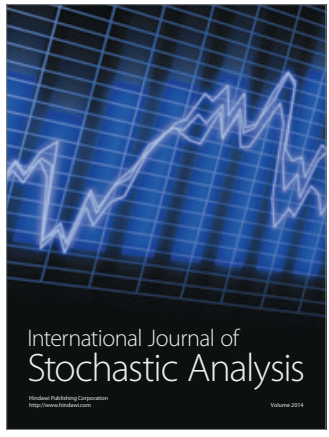

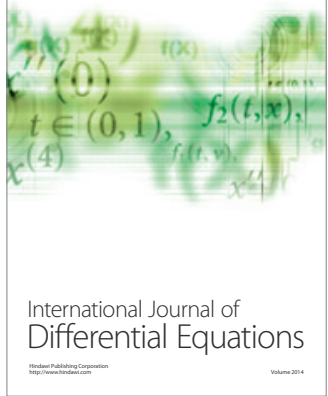
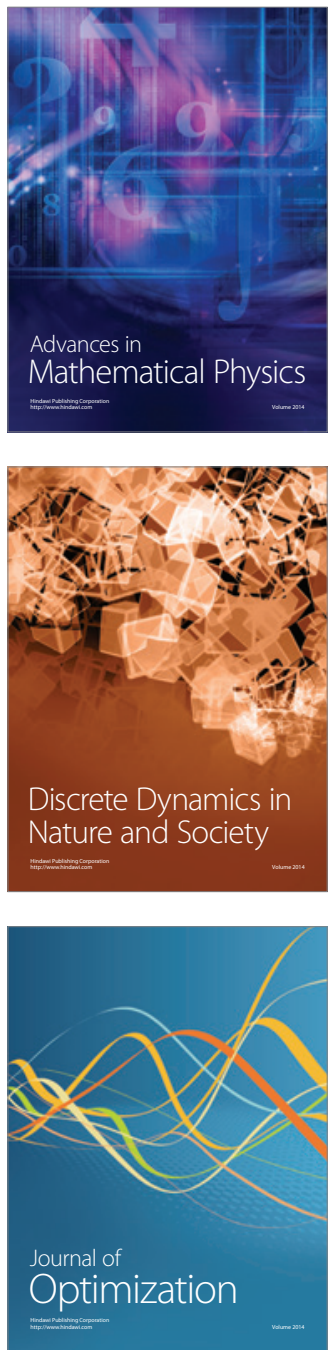